\title{
Exposure Assessment of Methyl Mercury from Consumption of Fish and Seafood in Peninsular Malaysia
}

Nurul Izzah Ahmad ( $\square$ nizzah.a@moh.gov.my)

Institute for Medical Research

Wan Rozita Wan Mahiyuddin

Institute for Medical Research

Wan Nurul Farah Wan Azmi

Institute for Medical Research

Ruzanaz Syafira Ruzman Azlee

Institute for Medical Research

Rafiza Shaharudin

Institute for Medical Research

Lokman Hakim Sulaiman

International Medical University

\section{Research Article}

Keywords: Methyl mercury, fish, seafood, exposure assessment, food safety, Malaysia

Posted Date: September 20th, 2021

DOI: https://doi.org/10.21203/rs.3.rs-808458/v1

License: (9) (i) This work is licensed under a Creative Commons Attribution 4.0 International License. Read Full License 


\section{Abstract}

The concentration of meHg in freshwater fish and seafood was investigated, as well as the consumption patterns of fish and seafood by different demographic groups (age, ethnicity, gender). A potential alarm for human-health hazards was also assessed, and the results were compared to the Provisional Tolerable Weekly Intakes (PTWIs) and the Hazard Quotient parameter (HQ). Results showed that meHg levels of 67 species ranged from 0.013 to $0.252 \mathrm{mg} / \mathrm{kg}$ of wet weight (WW) with significant differences between different fish and seafood groups $\left(\chi^{2}{ }_{K W}=49.09 ; p<0.001\right)$. Median concentrations of meHg in fish and seafood groups in descending orders: demersal fish $(0.1006 \mathrm{mg} / \mathrm{kg} \mathrm{WW})>$ pelagic fish $(0.0686 \mathrm{mg} / \mathrm{kg}$ $W W)>$ freshwater fish $0.045 \mathrm{mg} / \mathrm{kg} \mathrm{WW})>$ cephalopods $(0.0405 \mathrm{mg} / \mathrm{kg} \mathrm{WW})$ crustaceans $(0.0356 \mathrm{mg} / \mathrm{kg}$ $W W)$. Results revealed that older population $(>40$ years old $)$ consumed significantly $(p=0.000)$ more fish compared to younger generations and the elderly consumed the highest amounts of fish $(104.0 \pm 113.0$ $\mathrm{g} /$ day). The adolescents (10-17 years old) consumed more than double of amount for both cephalopod and crustacean compared to the older populations $(p<0.05)$. Malay ethnic $(96.1 \pm 99.6 \mathrm{~g} /$ day $)$ consumed significantly $(p=0.000)$ higher amounts of fish and seafood compared to other ethnicity, similarly to male subjects $(95.2 \pm 102 \mathrm{~g} /$ day; $p=0.026)$ when compared to the female $(86 \pm 96.3 \mathrm{~g} /$ day $)$. The estimated weekly intake (EWI) values showed results of below $1.6 \mu \mathrm{g} / \mathrm{kg} \mathrm{BW} /$ week, the tolerable levels recommended by the Joint FAO/WHO Expert Committee on Food Additives (JECFA) for all different demographic factors except for higher consumers at 75th percentile and above. Consumption of marine fish contributed to higher value of PTWI to all different demographic groups (the estimated weekly intake (EWI) range: $0.2988-0.6893 \mu \mathrm{g} / \mathrm{kg} \mathrm{BW} /$ week) but for the adolescents, where from the consumption of crustaceans $(0.3488 \mu \mathrm{g} / \mathrm{kg} \mathrm{BW} /$ week or $21.8 \%$ of PTWI) and cephalopods $(0.504 \mu \mathrm{g} / \mathrm{kg} \mathrm{BW} /$ week or $31.5 \%$ of PTWI). Results from this study also revealed the HQ value for overall consumption of fish and seafood by the adolescents and elderly exceeded one. This was contributed from the consumption of demersal fish and cephalopods, thus indicating the non-acceptable level of non-carcinogenic adverse health effects.

\section{Introduction}

Fish and seafood are a good source of energy and proteins, as well as key nutrients like minerals and vitamins, and they have a lot of health benefits (Mehouel et al 2019; Barone et al 2015). It also contains eicosapentaenoic acid (EPA), docosahexaenoic acid (DHA), and long-chain omega-3 polyunsaturated fatty acids (n-3 PUFAs), which are vital unsaturated fatty acids linked to excellent health (Barone et al 2015; Larsen et al. 2011; McManus et al. 2011; Sioen et al 2007). Medical research has shown that eating meat substitutes, fat fish or lean fish, and fish oil mixed with vegetables can improve fat consumption quality, lower calorie intake, and prevent lifestyle diseases, all of which are linked to a variety of health benefits, including maintaining healthy human hearts, brains, joints, and immune systems (Larsen et al. 2011; McManus et al. 2011).

Fish consumption, on the other hand, is the most prevalent way for humans to be exposed to mercury, which is released into the environment by both natural and human sources (Mehouel et al 2019; Castro- 
Gonalez \& Mendez-Armenta 2008). Mercury ( $\mathrm{Hg})$ exist mainly in different forms, an elemental mercury $(\mathrm{HgO})$, inorganic mercury $\left(\mathrm{Hg}^{+}, \mathrm{Hg}^{2+}\right)$ and organic mercury $\left(\mathrm{MeHg}^{+}, \mathrm{EtHg}^{+} \mathrm{PhHg}^{+}\right.$, and so on) (Mehouel et al 2019). In fish and seafood, it often found in an organic form of meHg as this compound is predominant (Mehouel et al 2019; Morgano et al. 2011; Burger 2009; Castro-Gonžalez \& Mendez-Armenta 2008; Myers \& Davidson 2000). It occurs at high percentage, ranges between 95 to $97 \%$ of total mercury ( $\mathrm{THg}$ ) when the compound accumulates in fish tissue. Hg linked to aquatic sediments is transformed into organic form by microbial activities through methylation and enzymatic processing, and it then enters the aquatic food chain, where it reaches its maximum concentration in predatory fish (Mehouel et al 2019; Clarkson et al. 2003). MeHg can be concentrated in fish either directly in the water or through food chain components (Castro-Gonzalez \& Mendez-Armenta 2008). The ingestion of fish contaminated with meHg has received considerable critical attention due to the adverse health outcomes of neurologic damage such as mental retardation, seizures, vision and hearing lost, delayed development, language disorder and memory loss as well as renal damage, reproductive disorders and damage in cardiovascular system (Mehouel et al 2019; Andrew et al 2016; Barone et al 2015). MeHg could potentially be one of the risk factors for infertility (Hsi et al 2016).

There are concerns about Malaysians being exposed to excessive levels of mercury as a result of eating fish and shellfish. Our earlier survey of people of various ethnicities in this country, conducted in the years 2008 to 2009, revealed that Malaysians had the second highest daily fish intake behind Japan among Asian nations, or placed fifth in the world (Ahmad et al 2016). The maximum permitted proportion of meHg in seafood was set at $0.5 \mathrm{mg} / \mathrm{kg}$ in Malaysian Food Regulation 1985 (Food Act 1983, (Act 281) and Regulations 2006) (International Law Book Services 2006), which is the same level as set by the joint FAO/WHO Expert Committee (FAO/WHO 2006). In view of the above, the FAO/WHO Expert Committee on Food Additives (JECFA) has established provisional tolerable weekly intake (PTWI) for meHg at 1.6 $\mu \mathrm{g} / \mathrm{Kg} / \mathrm{BW} /$ week. This is the safe level of intake or the maximum amount of a contaminant that be exposed to a person weekly over a lifetime without intolerable risk of health effects associated with the consumption of food (Wan Azmi et al 2019; Kuras et al 2017; WHO 2004). To estimate the potential of health risk due to exposure to the contaminant, the United State Environmental Protection Agency (USEPA) created the reference dose (RfD), an estimated of daily oral exposure of contaminant to the human population that is likely to be without considerable risk of harmful effects during a lifetime which is set at $0.0001 \mathrm{mg} / \mathrm{kg} / \mathrm{day}$ for $\mathrm{Hg}$ (Wan Azmi et al 2019; USEPA 2000)

Dietary intake data can be paired with data on meHg concentrations in edible tissues, consumption rates of fish or seafood, and the average body weight of studied or targeted populations to compute exposure to meHg indirectly (Suratno et al 2019; Annual et al 2018; You et al 2018;). While the potential for health risk was calculated utilising previously collected data such as exposure frequencies, duration of exposure, RfDs, and average exposure time (Wan Azmi et al 2019; Ouboter et al 2018; Bhupander \& Mukherjee 2011). Many research on metal pollution in fish and seafood have been conducted in Malaysia (Wan Azmi et al 2019; Low et al 2015; Alina et al 2012; Mok et al 2012; Kamaruzaman et al 2011; Agusa et al 2005), and a number of published data on THg or meHg levels in fish and seafood are 
available (Annual et al 2018; Ahmad et al. $2015^{\mathrm{ab}}$, Hajeb et al 2009). Many investigations found varying amounts of $\mathrm{THg}$ or meHg in a small number of fish and shellfish species gathered from specific locations around Malaysia. The results demonstrated that $\mathrm{THg}$ or $\mathrm{meHg}$ concentrations were low when compared to Malaysian Standards or JECFA recommended values. Several investigations found that some species of fish and shellfish seized from Malaysian markets have high THg or meHg contents, potentially putting consumers at risk (Ahmad et al. 2015 ab, Hajeb et al 2009, 2010; Agusa et al. 2005). Among those species are fork-tailed threadfin bream and big eye scads (Agusa et al 2005), long tail tuna, mackerel (Hajeb et al 2009), blue spotted stingray, honeycomb stingray, and John's snapper (Ahmad et al 2015a). Earlier study also reported on $48 \%$ of marine fish had $\mathrm{Hg}$ levels higher than the guideline value especially among carnivorous feeding (Agusa et al 2005).

In Malaysia, risk assessments on mercury have looked at the sources/locations, at-risk populations, fish species and families/groups, seafood preparation, and risk assessment methodologies (Agusa et al 2005, 2007; Hajeb et al 2009, 2011; Low et al 2015; Annual et al 2008; Wan Azmi et al 2019). The quantities of trace elements in fish gathered throughout Southeast Asia varied greatly, and the estimated daily intake (EDI) value was calculated higher due to the high consumption rate of seafood in Malaysia. The EDI for all specimens of fork-tailed threadfin bream and sharp-tooth job-fishes exceeded the guideline values and would indicate hazardous to the populations in this region (Agusa et al 2005, 2007). Current study showed that $14 \%$ of seafood had medium to high mercury concentrations with EWI higher than the PTWI for few species of bream, snapper, croaker, barramundi and tuna and the EWI values ranging from 2.1 to $4.0 \mu \mathrm{g} / \mathrm{kg} \mathrm{bw}$ (Annual et al 2018). The average Malaysian population was exposed to $1182 \mathrm{~g}$ per week, or $73.95 \%$ of the PTWI (Hajeb et al 2009), whereas the exposures for the fisherman families were as high as 2,332 $\mathrm{g}$ per week, or $145.8 \%$ of the PTWI (Hajeb et al 2011). Instead, the probabilistic estimation of reasonable exposure and non-carcinogenic risks associated with consumption of fresh water fish (red tilapia) at 95th percentile exposure revealed hazard quotient (HQ) and hazard index (HI) values below 0.2 and 1 , respectively, indicating that long-term consumption of this fish is likely to have negative consequences for Malaysians (Low et al 2015).

When assessing the risk of $\mathrm{Hg}$ or $\mathrm{meHg}$ from fish and shellfish diet, data from various other nations included more complete characteristics. There have been studies conducted to support regulatory analysis that rely on quantitative fish consumption estimates based on representative populations survey (von Stackelberg et al 2017; Lee et al 2006) or involved specific or broad range of seafood species consumed by population (Mehouel et al 2019; Budiyanto et al 2019; Barone et al 2015; Al-Mughairi et al 2013), collected from specific or different locations (Bhupander \& Mukherjee 2011; Uratno et al 2018) or wild and farm species (Chouvelon et al 2009). There are also studies involved vulnerable groups of pregnant women, children below 17 years old, women of child bearing age and other high-risk consumers (Stuchal et al 2019; You et al 2018; Kuras et al 2017; Juric et al 2017; Andrew et al 2016; Whyte et al 2009). Data from other sources also reported for common fish eaten or fish parts or organs for example fish muscle, liver, gills, kidney and others (Matos et al 2018; Zolfaghari 2018; Chen \& Chen 2006). There 
are also studies that estimate the risk of $\mathrm{Hg}$ contamination from seafood eating by analysing biological materials like blood and hair (You et al 2018; Ouboter et al 2018; Kuras et al 2017; Juric et al 2017).

This study updates information on the concentration of meHg in freshwater fish and seafood, including marine fish, cephalopods, and crustaceans, obtained at random from both fish landing ports and wholesale wet markets across Peninsular Malaysia. The study also looked at the consumption patterns of fish and seafood among Peninsular Malaysians of various age groups, ethnicities, and genders. Fish and seafood consumption for higher consumers was calculated at 75th percentile and above, and data were compared to the median consumption at 50 th percentile. A potential alarm for human health hazards were evaluated and results were compared to the PTWls and through the parameter of HQ.

\section{Methodology}

\section{Fish consumption survey}

Between February 2008 and May 2009, a household-based cross-sectional study was undertaken in Peninsular Malaysia, with data collected through face-to-face interviews using pre-designed questionnaires. Based on the National Household Sampling Frame (NHSF), Department of Statistics, Malaysia, a sampling frame made up of Enumeration Blocks (EBs) constructed for the 2000 Population and Housing Census was used to pick study subjects' household addresses (Department of Statistics Malaysia 2000). The sample size was calculated using data from a Selangor population consumption survey, which revealed that $16.2 \%$ of the adult population consumed fish $(153 \mathrm{~g} /$ person/day vs 944g/person/day total food) (Ahmad 2007). Factors from two separate places (urban and rural), three different ethnic groups (Malay, Chinese, and Indian), and two different age groups were used. A number of 2,496 subjects were required in order to obtain $95 \%$ confidence interval and $5 \%$ margin of error. Taken into account a $20 \%$ dropped-off rate, 2,996 subjects were identified from 1,500 household addresses received from the NHSF. In this study, a minimum of two adults and all adolescence ages 10 to 17 years were chosen from each family. The final count of 2,675 adult had completed the questionnaire. A number of 890 children/adolescent participated in the survey but only 484 completed the questionnaires.

The questionnaires were divided into two sections. The first portion was a self-administered questionnaire that included sections on socio-demographic information, fish consumption patterns, frequency of fish intake, and knowledge, perceptions, and practices related to fish eating. The three copies of 24-hour dietary diary forms made up the second half. In this portion, participants were instructed to keep track of what they ate and drank at each meal of the day. The interviewers were instructed how to read and understand the questionnaires and how to give instructions to the subjects. They were given a series of questionnaire tools which included pictures of serving dishes, fish commonly found in Malaysia and common household measure like standard measuring cups, bowls, ladles and spoons. The questionnaire was provided between the hours of 9:00 a.m. and 6:00 p.m., however interviewers were sometimes required to come at night if individuals were not at home during the day. Parents were asked to assist their children in filling out the 24-hour dietary diary forms and answering 
questions on the surveys. Interviewers were also re-checked all food recorded in dietary diary forms to verify types and amount of food consumed by subjects.

The portion weight of food was determined using the "Atlas Makanan: Saiz pertukaran dan Porsi" (Suzana et al 2002, 2009) and "Nutrient and composition of Malaysian foods" (Tee et al 1997). If the food consumed was not listed in all of these references, mean values were determined as the weight of that particular food from at least five different sources. The collections of the three days dietary diary were conducted during weekdays and weekends. Details on the calculation of sample size, questionnaires and interviews involved adults from Peninsular Malaysia for the whole study entitled "Seafood consumption survey in Peninsular Malaysia, 2008-2009" were as described elsewhere (Ahmad et al 2016), while similar information for survey that involved adolescence was reported recently (Ahmad et al 2019). Information on the demographic background of study subjects from both groups were presented in both published articles, accordingly (Ahmad et al 2016; 2019). The Medical Research and Ethics Committee (MREC) of the Ministry of Health Malaysia $(\mathrm{MOH})$ approved the project, which was funded by the $\mathrm{MOH}$. Informed consent and confidentiality were obtained from the subjects beforehand.

\section{Seafood collection and preparation}

Sampling was conducted from June to December 2009. Samples were obtained from six major Fisheries Development Authority of Malaysia (LKIM) fish landing complexes and five wholesale wet marketplaces across Peninsular Malaysia. During three trips to each location, a total of 394 seafood samples were gathered. Details on how to collect, prepare, process, and store fish can be found elsewhere (Ahmad et al. $\left.2015^{\mathrm{ab}}\right)$.

\section{Determination of total mercury concentrations in seafood}

In a microwave digestion device, a dried sample was digested (Multiwave 3000 - Anton Paar). Total mercury was measured using a Perkin Elmer Flow Injection Mercury System (FIMS) apparatus with a programmable sample dispenser and a cold vapour atomic absorption spectrometry (AAS) methodology, as described by Mohd Fairulnizal et al (1998). Analytical control was accompanied by analysis of reagent blanks and standard reference samples. Details on the analysis were described elsewhere (Ahmad et al. 2015 ab). It was essential to convert mercury contents in fish samples to wet basis values, and the quantity of moisture content was determined using Tee et al. (1997) and other sources (Nurnadia et al. 2011). Results were compared to the recommended guideline levels by the joint FAO/WHO Expert Committee on Food Additives (FAO/WHO 2006) and the Malaysian Food Regulation 1985 (Food Act 1983, (Act 281), and Regulations 2006), under the Fourteenth Schedule of Regulation 38, the level at 0.5 $\mathrm{mg} / \mathrm{kg} \mathrm{meHg}$ in fish and seafood.

\section{Health risk assessment of mercury from seafood consumption}

\section{Estimated Weekly Intake (EWI) and Maximum Safe Weekly Consumption (MSWC)}


The EWI of total $\mathrm{Hg}$ and/or meHg from consumption of seafood can be calculated by multiplying the total $\mathrm{Hg}$ and/or meHg contamination levels in seafood with the consumption levels per week dividing by the average body weight of the population by age group. The equation is as shown below:

$\mathrm{EWI}=\underline{\text { Concentration of } \mathrm{meHg}}(\underline{\mathrm{mg}} / \mathrm{Kg} \underline{\mathrm{WW}}) \mathbf{x}$ weekly consumption $(\mathrm{g})$.

Body weight $(\mathrm{kg})$

The typical adult body weight varies between 55 and $62 \mathrm{~kg}$ depending on demographic factors, while the average adolescent body weight is $45 \mathrm{~kg}$ (Ahmad et al 2016; 2019). The JECFA has defined a PTWI of 1.6 $\mathrm{g} / \mathrm{kg}$ bodyweight/week for inorganic MeHg (WHO 2011).

MSWC to reach the PTWI for Malaysian population at different socio-demographic characteristics was also estimated. The MSWC was calculated using the PTWI ( $\mathrm{g} / \mathrm{kg}$ body weight/week) value of $1.6 \mathrm{~g} / \mathrm{kg}$ body weight/week multiplied by body weight for each population group and divided by total $\mathrm{Hg}$ and/or meHg concentrations.

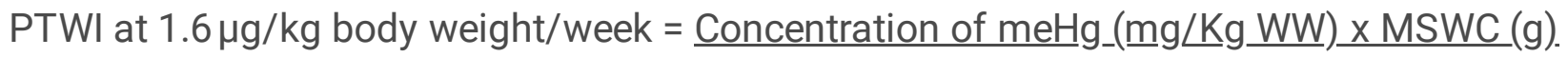

Body weight $(\mathrm{kg})$

\section{Hazard Quotient (HQ)}

Risk assessment is a tool to estimate the probability of health effects due to exposure to the hazard, in which this study is the exposure through consumption of fish. The oral reference dosage (RfDs) for mercury was set at 1 x 10-4 (mg/kg-day) by the USEPA (Risk Information System (IRIS) (USEPA 2000). The HQ meHg was estimated using the equation below (Wan Azmi et al 2019):

$\mathrm{HQ}=\underline{\mathrm{EF} \times E D \times F I R \times C} \times 10^{-3}$

$\mathrm{RfD} \times \mathrm{BW} \times \mathrm{AT}$

where $\mathrm{HQ}$ is chemical-specific Hazard Quotient; EF is the exposure frequency (350 days/year); ED is the duration of human exposure for children and adults is 6 and 30 years, respectively; FIR is the seafood ingestion rate (based on total intake per day in gram by different groups of population); $C$ is the metal concentration in the muscle of fishes ( $\mathrm{mg} / \mathrm{kg}$ wet weight); RfD is the oral reference dose (IRIS, USEPA); BW is the average body weight of population group ( 55 to $62 \mathrm{~kg}$ for adult, $45 \mathrm{~kg}$ for adolescent and $60 \mathrm{~kg}$ for total population) and AT is average time of human exposure to non-carcinogenic (ED x 365 days).

Target hazard is a ratio of the determined dose of a contaminant to oral reference dose considered detrimental. $\mathrm{HQ}$ values were more than or equal to 1 , it is assumed that the intake of meHg through the consumption of fish and seafood poses a potential non-carcinogenic health risk to the exposed population. 


\section{Statistical analysis}

IBM SPSS Statistics 26 was used to analyze the data. Before analyzing the THg data, it was cleaned and examined for discrepancies. The demographic features of different categories and groups were keyed in using data from a dietary survey. After completing the data entry, it was checked for any discrepancies, such as coding numbers, typo errors, and so on. At first, descriptive statistics were used to assess data normality using the one-sample Kolmogorov-Smirnov test, and/or the skewness of descriptive statistics was regulated between -1 and +1 , whichever was true. Because of the outliers, the descriptive statistical analysis revealed that both groups of data were not regularly distributed. As a result, non-parametric statistics were employed. The medians and interquartile range were calculated The Mann-Whitney $U$ and Kruskall-Wallis tests were used to analyze differences across groups. A level of significance at 0.05 is set to determine the result is statistically significant. Significance values have been adjusted by the Bonferroni correction for multiple tests. Higher consumers were chosen among participants who consumed fish and seafood at the 75th, 90th, and 95th percentiles, and their consumption was compared to the median consumption at the 50 th percentile.

\section{Results}

\section{$\mathrm{MeHg}$ in fish and seafood}

$\mathrm{THg}$ and $\mathrm{meHg}$ (median $\pm \mathrm{IQR}$ ) concentrations in marine and freshwater fish from fish landing ports and the wholesale markets in Peninsular Malaysia are summarized in Table 1. MeHg levels of 67 fish and seafood species ranged from 0.013 to $0.252 \mathrm{mg} / \mathrm{kg}$ of wet weight. Fish and seafood groups were comprised of 8 species of cephalopods, 12 species of crustaceans, 23 species of demersal fish, 1 species of freshwater fish and 23 species of pelagic fish. Significant variations of meHg levels exist in different fish and seafood groups $\left(\chi^{2}{ }_{k W}=137.486 ; p<0.001\right)$. Among marine fish, the median for meHg levels was higher ( $>0.1 \mathrm{mg} / \mathrm{kg} \mathrm{WW}$ ) in two species of pelagic fish (Selar boops and Sarda orientalis) and eight species of demersal fish (Lutjanus argentimaculatus, Lutjanus russellii, Lates calcarifer, Psammoperca waigiensis, Dasyatis zugei, Nemipterus japonicus, Nemipterus furcosus and Nemipterus nematophorus). While for cephalopods and crustaceans as well as the freshwater catfish, meHg levels were nearly half compared to the marine fish at $0.045,0.046,0.066 \mathrm{mg} / \mathrm{kg}$ WW, respectively. Pairwise comparisons using Bonferroni correction of meHg levels in fish and seafood groups showed significant differences between pelagic fish and the other three groups of demersal fish $(p=0.000)$; crustacean $(p=0.000)$ and cephalopod $(p=0.000)$. There were also significant differences between demersal fish and crustaceans $(p=0.000)$, cephalopod $(p=0.000)$ and freshwater fish $(p=0.048)$. Median concentrations of meHg in fish and seafood groups in descending orders: demersal fish $(0.1006 \mathrm{mg} / \mathrm{kg} \mathrm{WW})>$ pelagic fish $(0.0686$ $\mathrm{mg} / \mathrm{kg} \mathrm{WW})>$ freshwater fish $0.045 \mathrm{mg} / \mathrm{kg} \mathrm{WW})>$ cephalopods $(0.0405 \mathrm{mg} / \mathrm{kg} \mathrm{WW})$ crustaceans $(0.0356$ $\mathrm{mg} / \mathrm{kg} \mathrm{WW}$ ). The calculation of median concentrations of meHg per species by means of the percentage to $\mathrm{THg}$ at $93 \%, 81 \%$ and $50 \%$ for fish, cephalopods and crustaceans, respectively (Annual et al 2018). 
These resulted showed that none of meHg levels in fish and seafood groups exceeded both the national and international guidelines. 
Table 1

Total $\mathrm{Hg}$ and $\mathrm{meHg}(\mathrm{mg} / \mathrm{kg} \mathrm{WW}$ ) levels in fish/seafood from the LKIM Complexes and wholesale market in Peninsular Malaysia

\begin{tabular}{|c|c|c|c|c|c|c|c|}
\hline Common name & Species & n & $\begin{array}{l}\text { total } \mathrm{Hg} \\
\text { (DW) }\end{array}$ & IQR & $\begin{array}{l}\text { \#MC } \\
(\%)\end{array}$ & $\begin{array}{l}\text { total } \\
\mathrm{Hg} \\
\text { (WW) }\end{array}$ & $\begin{array}{l}\text { *meHg } \\
\text { (WW) }\end{array}$ \\
\hline \multicolumn{8}{|l|}{ Pelagic fish } \\
\hline Yellowstripe scad & $\begin{array}{l}\text { Selaroides } \\
\text { leptolepis }\end{array}$ & 10 & 0.252 & 0.125 & 79.5 & 0.0517 & 0.0480 \\
\hline Oxeye scad & Selar boops & 3 & 0.555 & - & 78.2 & 0.1210 & 0.1125 \\
\hline Bigeye scad & $\begin{array}{l}\text { Selar } \\
\text { crumenopthalmus }\end{array}$ & 1 & 0.298 & - & 78.8 & 0.0632 & 0.0588 \\
\hline Yellowtail scad & Atule mate & 4 & 0.458 & 0.304 & 76.8 & 0.1063 & 0.0988 \\
\hline Bigeye trevally & $\begin{array}{l}\text { Caranx } \\
\text { sexfasciatus }\end{array}$ & 1 & 0.293 & - & 76.8 & 0.0680 & 0.0632 \\
\hline Greater amberjack & Seriola dumerili & 1 & 0.203 & - & 84.7 & 0.0311 & 0.0289 \\
\hline Redtail scad & $\begin{array}{l}\text { Decapterus } \\
\text { kurroides }\end{array}$ & 2 & 0.272 & 0.263 & 74.7 & 0.0688 & 0.0640 \\
\hline Round scad & $\begin{array}{l}\text { Decapterus } \\
\text { muruadsi }\end{array}$ & 7 & 0.317 & 0.171 & 77.4 & 0.0716 & 0.0666 \\
\hline Slender scad & Decapterus russelli & 4 & 0.195 & 0.108 & 74.7 & 0.0493 & 0.0459 \\
\hline Shortfin scad & $\begin{array}{l}\text { Decapterus } \\
\text { macrosoma }\end{array}$ & 1 & 0.354 & - & 74.7 & 0.0896 & 0.0833 \\
\hline Torpedo scad & Megalaspis cordyla & 17 & 0.319 & 0.198 & 74.8 & 0.0804 & 0.0748 \\
\hline Black pomfret & $\begin{array}{l}\text { Parastromateus } \\
\text { niger }\end{array}$ & 8 & 0.242 & 0.121 & 76.5 & 0.0569 & 0.0529 \\
\hline Indian mackerel & $\begin{array}{l}\text { Rastrelliger } \\
\text { kanagurta }\end{array}$ & 9 & 0.18 & 0.066 & 73.1 & 0.0484 & 0.0450 \\
\hline Faughn's mackerel & Rastrelliger faughni & 3 & 0.357 & 0.246 & 77.9 & 0.0789 & 0.0734 \\
\hline $\begin{array}{l}\text { Indo-Pacific } \\
\text { mackerel }\end{array}$ & $\begin{array}{l}\text { Rastrelliger } \\
\text { brachysoma }\end{array}$ & 3 & 0.261 & - & 78.9 & 0.0551 & 0.0512 \\
\hline Slimmy mackerel & $\begin{array}{l}\text { Scomber } \\
\text { australasicus }\end{array}$ & 10 & 0.269 & 0.065 & 77.7 & 0.0600 & 0.0558 \\
\hline $\begin{array}{l}\text { Indo-Pacific king } \\
\text { mackerel }\end{array}$ & $\begin{array}{l}\text { Scomberomorus } \\
\text { guttatus }\end{array}$ & 9 & 0.262 & 0.355 & 75.9 & 0.0631 & 0.0587 \\
\hline $\begin{array}{l}\text { Narrowbarred } \\
\text { Spanish mackerel }\end{array}$ & $\begin{array}{l}\text { Scomberomorus } \\
\text { commerson }\end{array}$ & 9 & 0.368 & 0.953 & 75.5 & 0.0902 & 0.0838 \\
\hline
\end{tabular}




\begin{tabular}{|c|c|c|c|c|c|c|c|}
\hline Common name & Species & $\mathbf{n}$ & $\begin{array}{l}\text { total } \mathrm{Hg} \\
\text { (DW) }\end{array}$ & IQR & $\begin{array}{l}\text { \#MC } \\
(\%)\end{array}$ & $\begin{array}{l}\text { total } \\
\mathrm{Hg} \\
(\mathrm{WW})\end{array}$ & $\begin{array}{l}{ }^{*} \mathrm{meHg} \\
(\mathrm{WW})\end{array}$ \\
\hline Dogtooth tuna & $\begin{array}{l}\text { Gymnosarda } \\
\text { unicolor }\end{array}$ & 9 & 0.342 & 0.456 & 74.5 & 0.0872 & 0.0811 \\
\hline striped bonito & Sarda orientalis & 6 & 0.543 & 1.048 & 76.9 & 0.1254 & 0.1167 \\
\hline Longtail tuna & Thunnus tonggol & 7 & 0.358 & 0.173 & 71 & 0.1038 & 0.0966 \\
\hline Frigate tuna & $\begin{array}{l}\text { Auxis thazard } \\
\text { thazard }\end{array}$ & 2 & 0.237 & - & 76.8 & 0.0550 & 0.0511 \\
\hline \multirow[t]{2}{*}{ Kawakawa } & Euthymus affinis & 2 & 0.289 & - & 75.2 & 0.0717 & 0.0667 \\
\hline & Total & 128 & 0.31 & 0.17 & 76.6 & 0.0738 & 0.0686 \\
\hline \multicolumn{8}{|l|}{ Table 1: continue } \\
\hline Common name & Species & $\mathrm{n}$ & $\begin{array}{l}\text { total } \mathrm{Hg} \\
\text { (DW) }\end{array}$ & IQR & $\begin{array}{l}{ }^{\# M C} \\
(\%)\end{array}$ & $\begin{array}{l}\text { total } \\
\mathrm{Hg} \\
\text { (WW) }\end{array}$ & $\begin{array}{l}{ }^{*} \mathrm{meHg} \\
(\mathrm{WW})\end{array}$ \\
\hline \multicolumn{8}{|l|}{ Demersal fish } \\
\hline $\begin{array}{l}\text { Mangrove red } \\
\text { snapper }\end{array}$ & $\begin{array}{l}\text { Lutjanus } \\
\text { argentimaculatus }\end{array}$ & 3 & 0.856 & - & 75.8 & 0.2072 & 0.1927 \\
\hline $\begin{array}{l}\text { Humpback red } \\
\text { snapper }\end{array}$ & Lutjanus gibbus & 1 & 0.436 & - & 82.1 & 0.0780 & 0.0726 \\
\hline Emperor red snapper & Lutjanus sebae & 10 & 0.334 & 0.516 & 80.7 & 0.0645 & 0.0599 \\
\hline $\begin{array}{l}\text { Malabar blood } \\
\text { snapper }\end{array}$ & $\begin{array}{l}\text { Lutjanus } \\
\text { malabaricus }\end{array}$ & 3 & 0.413 & 0.366 & 80.9 & 0.0789 & 0.0734 \\
\hline John's snapper & Lutjanus russellii & 4 & 1.366 & - & 80.2 & 0.2705 & 0.2515 \\
\hline Giant sea perch & Lates calcarifer & 7 & 0.537 & 0.436 & 78.1 & 0.1176 & 0.1094 \\
\hline Waigeu sea perch & $\begin{array}{l}\text { Psammoperca } \\
\text { waigiensis }\end{array}$ & 4 & 0.532 & 0.165 & 79.1 & 0.1112 & 0.1034 \\
\hline Sharpnose stingray & Himantura gerrardi & 8 & 0.384 & 0.741 & 79.1 & 0.0803 & 0.0746 \\
\hline Bluespotted stingray & Neotrygon kuhlii & 6 & 0.492 & 1.251 & 82 & 0.0886 & 0.0824 \\
\hline Pale-edged stingray & Dasyatis zugei & 4 & 0.548 & 0.509 & 76.1 & 0.1310 & 0.1218 \\
\hline Honeycomb stingray & Himantura uarnak & 2 & 0.425 & - & 79.2 & 0.0884 & 0.0822 \\
\hline Reeve's croaker & Chrysochir aureus & 3 & 0.498 & - & 80.6 & 0.0966 & 0.0898 \\
\hline Tigertooth croaker & Otolithoides ruber & 5 & 0.421 & 0.423 & 79.9 & 0.0846 & 0.0787 \\
\hline Soldier croaker & Nibea soldado & 11 & 0.424 & 0.132 & 76.8 & 0.0984 & 0.0915 \\
\hline
\end{tabular}




\begin{tabular}{|c|c|c|c|c|c|c|c|}
\hline Common name & Species & $\mathbf{n}$ & $\begin{array}{l}\text { total } \mathrm{Hg} \\
\text { (DW) }\end{array}$ & IQR & $\begin{array}{l}{ }^{\# M C} \\
(\%)\end{array}$ & $\begin{array}{l}\text { total } \\
\mathrm{Hg} \\
(\mathrm{WW})\end{array}$ & $\begin{array}{l}{ }^{*} \mathrm{meHg} \\
\text { (WW) }\end{array}$ \\
\hline Bronze croaker & $\begin{array}{l}\text { Otolithoides } \\
\text { biauritus }\end{array}$ & 1 & 0.069 & - & 79.9 & 0.0139 & 0.0129 \\
\hline $\begin{array}{l}\text { Yellowbelly threadfin } \\
\text { bream }\end{array}$ & $\begin{array}{l}\text { Nemipterus } \\
\text { bathybius }\end{array}$ & 5 & 0.383 & 0.328 & 76.9 & 0.0885 & 0.0823 \\
\hline $\begin{array}{l}\text { Japanese threadfin } \\
\text { bream }\end{array}$ & $\begin{array}{l}\text { Nemipterus } \\
\text { japonicus }\end{array}$ & 9 & 0.464 & 0.724 & 76.9 & 0.1072 & 0.0997 \\
\hline $\begin{array}{l}\text { Forktail threadfin } \\
\text { bream }\end{array}$ & $\begin{array}{l}\text { Nemipterus } \\
\text { furcosus }\end{array}$ & 3 & 0.642 & - & 79.2 & 0.1335 & 0.1242 \\
\hline Threadfin bream & $\begin{array}{l}\text { Nemipterus } \\
\text { thosaporni }\end{array}$ & 2 & 0.57 & 0.659 & 82.4 & 0.1003 & 0.0933 \\
\hline $\begin{array}{l}\text { Fivelined threadfin } \\
\text { bream }\end{array}$ & $\begin{array}{l}\text { Nemipterus } \\
\text { tambuloides }\end{array}$ & 2 & 0.426 & - & 78.1 & 0.0933 & 0.0868 \\
\hline $\begin{array}{l}\text { Doublewhip } \\
\text { threadfin bream }\end{array}$ & $\begin{array}{l}\text { Nemipterus } \\
\text { nematophorus }\end{array}$ & 2 & 1.211 & - & 80.4 & 0.2374 & 0.2207 \\
\hline \multicolumn{8}{|l|}{ Table 1: continue } \\
\hline Common name & Species & $\mathrm{n}$ & $\begin{array}{l}\text { total } \mathrm{Hg} \\
\text { (DW) }\end{array}$ & IQR & $\begin{array}{l}{ }^{\#} \mathrm{MC} \\
(\%)\end{array}$ & $\begin{array}{l}\text { total } \\
\mathrm{Hg} \\
(\mathrm{WW})\end{array}$ & $\begin{array}{l}{ }^{*} \mathrm{meHg} \\
(\mathrm{WW})\end{array}$ \\
\hline $\begin{array}{l}\text { Red filament } \\
\text { threadfin bream }\end{array}$ & $\begin{array}{l}\text { Nemipterus } \\
\text { marginatus }\end{array}$ & 2 & 0.244 & - & 76.2 & 0.0581 & 0.0540 \\
\hline \multirow[t]{3}{*}{$\begin{array}{l}\text { Redspine threadfin } \\
\text { bream }\end{array}$} & $\begin{array}{l}\text { Nemipterus } \\
\text { nemurus }\end{array}$ & 1 & 0.298 & - & 79.5 & 0.0611 & 0.0568 \\
\hline & Total & 98 & 0.46 & 0.41 & 79.1 & 0.1082 & 0.1006 \\
\hline & Total marine fish & 226 & 0.42 & 0.40 & 77.9 & 0.0910 & 0.0846 \\
\hline \multicolumn{8}{|l|}{ Freshwater fish } \\
\hline Catfish & Clarias batrachus & 9 & 0.334 & 0.325 & 77.1 & 0.0490 & 0.0450 \\
\hline \multicolumn{8}{|l|}{ Cephalopods } \\
\hline Golden cuttlefish & Sephia esculenta & 6 & 0.257 & 0.11 & 81.4 & 0.0478 & 0.0387 \\
\hline Indian squid & Sepia phuruonis & 10 & 0.199 & 0.16 & 81.4 & 0.0614 & 0.0497 \\
\hline Little squid & Loligo duvaucelli & 4 & 0.249 & - & 81.4 & 0.0370 & 0.0300 \\
\hline Mitre squid & Loligo uyii & 7 & 0.275 & 0.12 & 81.4 & 0.0463 & 0.0375 \\
\hline Old women octopus & Loligo chinensis & 1 & 0.208 & - & 81.4 & 0.0512 & 0.0414 \\
\hline Pharoah cuttlefish & Loligo sibogae & 2 & 0.33 & - & 81.4 & 0.0677 & 0.0548 \\
\hline
\end{tabular}




\begin{tabular}{|c|c|c|c|c|c|c|c|}
\hline Common name & Species & $\mathbf{n}$ & $\begin{array}{l}\text { total } \mathrm{Hg} \\
\text { (DW) }\end{array}$ & IQR & $\begin{array}{l}\text { \#MC } \\
(\%)\end{array}$ & $\begin{array}{l}\text { total } \\
\mathrm{Hg} \\
(\mathrm{WW})\end{array}$ & $\begin{array}{l}{ }^{*} \mathrm{meHg} \\
(\mathrm{WW})\end{array}$ \\
\hline Sibogae squid & Loligo edulis & 6 & 0.364 & 0.51 & 81.4 & 0.0497 & 0.0402 \\
\hline \multirow[t]{2}{*}{ Sword tip squid } & Cistopus indicus & 9 & 0.267 & 0.28 & 81.4 & 0.0387 & 0.0313 \\
\hline & Total & 45 & 0.25 & 0.13 & 81.4 & 0.0500 & 0.0405 \\
\hline \multicolumn{8}{|l|}{ Crustaceans } \\
\hline Banana prawn & $\begin{array}{l}\text { Penaeus } \\
\text { merguiensis }\end{array}$ & 7 & 0.277 & 0.10 & 80.5 & 0.0540 & 0.0270 \\
\hline Giant tiger prawn & $\begin{array}{l}\text { Penaeus monodon } \\
\mathrm{S}\end{array}$ & 2 & 0.399 & - & 80.5 & 0.0778 & 0.0389 \\
\hline \multirow[t]{2}{*}{ Greasyback shrimp } & $\begin{array}{l}\text { Penaeus } \\
\text { semisulcatus }\end{array}$ & 3 & 0.251 & - & 80.5 & 0.0532 & 0.0266 \\
\hline & & & & & \multicolumn{3}{|c|}{ continue } \\
\hline \multicolumn{8}{|l|}{ Table 1: continue } \\
\hline Common name & Species & $\mathrm{n}$ & $\begin{array}{l}\text { total } \mathrm{Hg} \\
\text { (DW) }\end{array}$ & IQR & $\begin{array}{l}{ }^{\# M C} \\
(\%)\end{array}$ & $\begin{array}{l}\text { total } \\
\mathrm{Hg} \\
\text { (WW) }\end{array}$ & $\begin{array}{l}{ }^{*} \mathrm{meHg} \\
(\mathrm{WW})\end{array}$ \\
\hline Green tiger prawn & Penaeus indicus & 2 & 0.273 & - & 80.5 & 0.0538 & 0.0269 \\
\hline Indian white prawn & Penaeus japonicus & 8 & 0.276 & 0.13 & 80.5 & 0.2650 & 0.1325 \\
\hline Kuruma prawn & $\begin{array}{l}\text { Penaeus } \\
\text { latisulcatus }\end{array}$ & 1 & 1.359 & - & 80.5 & 0.0710 & 0.0355 \\
\hline Pink shrimp & Metapenaeus ensis & 4 & 0.28 & 0.50 & 80.5 & 0.0489 & 0.0245 \\
\hline Rainbow shrimp & $\begin{array}{l}\text { Metapenaeus } \\
\text { affinis }\end{array}$ & 4 & 0.242 & 0.25 & 80.5 & 0.0546 & 0.0273 \\
\hline Sand velvet shrimp & $\begin{array}{l}\text { Parapenaeopsis } \\
\text { sculptilis }\end{array}$ & 9 & 0.269 & 0.08 & 80.5 & 0.0472 & 0.0236 \\
\hline Spear shrimp & $\begin{array}{l}\text { Metapenaeopsis } \\
\text { barbata }\end{array}$ & 3 & 0.176 & - & 80.5 & 0.0525 & 0.0262 \\
\hline Western king prawn & $\begin{array}{l}\text { Parapenaeospsis } \\
\text { hardwickii }\end{array}$ & 5 & 0.364 & 0.17 & 80.5 & 0.0343 & 0.0172 \\
\hline \multirow[t]{3}{*}{ Yellow shrimp } & $\begin{array}{l}\text { Metapenaeus } \\
\text { brevicornis }\end{array}$ & 4 & 0.215 & 0.05 & 80.5 & 0.0419 & 0.0210 \\
\hline & Total & 52 & 0.272 & 0.15 & 80.5 & 0.0712 & 0.0356 \\
\hline & Overall & 405 & 0.06 & 0.05 & 78.74 & 0.0610 & 0.0305 \\
\hline
\end{tabular}




\begin{tabular}{|c|c|c|c|c|c|c|}
\hline Common name & Species & $\mathrm{n}$ & $\begin{array}{l}\text { total } \mathrm{Hg} \\
\text { (DW) }\end{array}$ & $\begin{array}{l}\text { \#MC } \\
(\%)\end{array}$ & $\begin{array}{l}\text { total } \\
\mathrm{Hg} \\
\text { (WW) }\end{array}$ & $\begin{array}{l}\text { *meHg } \\
(\mathrm{WW})\end{array}$ \\
\hline \multicolumn{7}{|c|}{ Total Hg in median \pm IQR; } \\
\hline \multicolumn{7}{|l|}{ DW - dry weight; } \\
\hline \multicolumn{7}{|c|}{ IQR - interquartile range; } \\
\hline \multicolumn{7}{|c|}{ MC - moisture content; } \\
\hline \multicolumn{7}{|c|}{ \#MC content was based on the works by Tee et al. (1997) and Nurnadia et al. (2011). } \\
\hline \multicolumn{7}{|c|}{$\begin{array}{l}\text { WW - wet weight; conversion of DW mercury concentrations in fish samples to WW were by means } \\
\text { formula: } D W=W W \times(100 / 100 M C) \text {. Details on total mercury concentrations in seafood is referred to } \\
\text { Ahmad et al }\left(2015^{\mathrm{ab}}\right) \text {; }\end{array}$} \\
\hline \multicolumn{7}{|c|}{$\begin{array}{l}{ }^{*} \text { calculation of meHg concentrations were based on mean percentage of methylmercury to total } \\
\text { mercury at } 93 \% \text { for fish, } 81 \% \text { for cephalopods and } 50 \% \text { for crustaceans (Annual et al 2018). }\end{array}$} \\
\hline \multicolumn{7}{|c|}{$\begin{array}{l}\text { Comparison of meHg levels for different fish/seafood groups: } \chi^{2}{ }_{K W}=49.090, p=0.000, N=405 \text {, } \\
\text { Median }=0.061 \pm 0.050 \mathrm{mg} / \mathrm{kg} \mathrm{WW} \text {. }\end{array}$} \\
\hline
\end{tabular}

\section{Fish And Seafood Consumption At Different Demographic Background}

Fish and seafood consumption (g/day/person) (median \pm IQR) by population in Peninsular Malaysia at different demographic categories were shown in Table $2-4$. There are significant differences $(p<0.05)$ between the consumption of seafood among different age groups for all fish and seafood categories except for freshwater fish (Table 2). Overall, the results revealed that older population (>40years old) consumed significantly $(p=0.000)$ more fish compared to younger generations $(<40$ years old $)$. The elderly consumed the highest amounts of fish $(104.0 \pm 113.0 \mathrm{~g} /$ day $)$, but the difference is not significant when compared to the next age group of 41 to 60 years old $(95.8 \pm 99.8 \mathrm{~g} /$ day). Nevertheless, the differences are significant $(p<0.05)$ when compared to the other two age groups, the adolescents (10 to 17 years old $)(84.9 \pm 104.1 \mathrm{~g} /$ day) and the young adults (18 to 40 years old) $(82.0 \pm 89.1 \mathrm{~g} /$ day $)$. In addition, the adolescents (10-17 years old) consumed about half amounts of marine fish $(26.0 \pm 30.6$ $\mathrm{g} /$ day) compared to the older population and the differences are significant at $\mathrm{p}=0.000$. Conversely, the amount they consumed were more than double for both cephalopod $(80.0 \pm 90.0 \mathrm{~g} /$ day) and crustacean $(63.0 \pm 65.0 \mathrm{~g} /$ day $)$ compared to the consumption by the older populations $(p<0.05)$. 
Table 2

Freshwater fish and seafood consumption ( $\mathrm{g} /$ day/person) (median $\pm \mathrm{IQR}$ ) by population in Peninsular Malaysia at different age categories

\begin{tabular}{|c|c|c|c|c|c|}
\hline \multirow[t]{3}{*}{ Food category } & \multicolumn{4}{|c|}{ Age by category } & \multirow[t]{3}{*}{${ }^{\# p-v a l u e ~}$} \\
\hline & 10-17yrs & $18-40 y r s$ & 41-60yrs & $\geq 61 \mathrm{yrs}$ & \\
\hline & $(n=653)$ & $(n-1,209)$ & $(n=1073)$ & $(n=422)$ & \\
\hline Pelagic fish & $22.0 \pm 32.7^{a}$ & $44.0 \pm 60.7^{b}$ & $48.7 \pm 66.4^{\mathrm{bc}}$ & $48.3 \pm 66.8^{\mathrm{bc}}$ & 0.000 \\
\hline Demersal fish & $30.5 \pm 26.0^{\mathrm{a}}$ & $46.0 \pm 57.7^{a}$ & $35.0 \pm 46.5^{\mathrm{b}}$ & $52.0 \pm 71.8^{b}$ & 0.017 \\
\hline Total marine fish & $26.0 \pm 30.6^{a}$ & $50.0 \pm 77.0^{b}$ & $60.0 \pm 77.8^{\mathrm{bc}}$ & $66.0 \pm 100.7^{c}$ & 0.000 \\
\hline Total freshwater fish & 38.339 .3 & $35.3 \pm 45.4$ & $36.7 \pm 47.0$ & $36.7 \pm 77.3$ & 0.790 \\
\hline Cephalopods & $80.0 \pm 90.0^{\mathrm{a}}$ & $30.0 \pm 37.0^{b}$ & $40.0 \pm 31.9^{b c}$ & $29.8 \pm 31.9^{b c}$ & 0.000 \\
\hline Crustaceans & $63.0 \pm 65.0^{\mathrm{a}}$ & $13.3 \pm 26.7^{b}$ & $21.0 \pm 28.9^{b c}$ & $13.3 \pm 22.9^{\mathrm{bc}}$ & 0.000 \\
\hline Overall consumption & $84.9 \pm 104.1^{\mathrm{a}}$ & $82.0 \pm 89.1^{\mathrm{a}}$ & $95.8 \pm 99.8^{b}$ & $104.0 \pm 113.0^{\mathrm{bc}}$ & 0.000 \\
\hline \multicolumn{6}{|c|}{ Age categories:10-17yrs-adolescents,18-40yrs-young adults, 41-60yrs-older adults, $\geq 61 \mathrm{yrs}$-elderly; } \\
\hline \multicolumn{6}{|c|}{ IQR - Inter-quartile Range } \\
\hline \multicolumn{6}{|c|}{${ }^{{ }^{K} \text { Kruskal-Wallis test were applied }}$} \\
\hline
\end{tabular}

Table 3: Freshwater fish and seafood consumption (g/day/person) (median \pm IQR) by different ethnicity in Peninsular Malaysia 


\begin{tabular}{|lllll|}
\hline Food category & \multicolumn{2}{l}{ Ethnicity } & \multirow{2}{*}{ \# $\mathrm{p}$-value } \\
\cline { 2 - 4 } & $\begin{array}{l}\text { Malays } \\
(\mathrm{n}=2,592)\end{array}$ & $\begin{array}{l}\text { Chinese } \\
(\mathrm{n}=457)\end{array}$ & $\begin{array}{l}\text { Indians } \\
(\mathrm{n}=270)\end{array}$ & \\
\hline Pelagic fish & $58.7 \pm 64.0^{\mathrm{a}}$ & $55.3 \pm 46.5^{\mathrm{a}}$ & $20.0 \pm 79.0^{\mathrm{c}}$ & 0.009 \\
\hline Demersal fish & $46.0 \pm 49.0$ & $37.3 \pm 53.2$ & $42.3 \pm 37.6$ & 0.763 \\
\hline Total marine fish & $69.5 \pm 73.3^{\mathrm{a}}$ & $45.0 \pm 82.7^{\mathrm{ab}}$ & $73.3 \mathrm{~b}^{\mathrm{c}}$ & 0.046 \\
\hline Total freshwater fish & $36.7 \pm 45.7$ & $42.7 \pm 30.8$ & 44.0 & 0.532 \\
\hline Cephalopods & $47.8 \pm 42.0$ & $53.3 \pm 0.0$ & $26.7 \pm 28.3$ & 0.741 \\
\hline Crustaceans & $21.3 \pm 20.7$ & $3.8 \pm 0.0$ & $70.0 \pm 36.5$ & 0.908 \\
\hline Overall consumption & $96.1 \pm 99.6^{\mathrm{a}}$ & $66.0 \pm 88.0^{\mathrm{b}}$ & $60.0 \pm 64.5^{\mathrm{bc}}$ & 0.000 \\
\hline IQR - Inter-quartile Range & & & & \\
\hline \#Kruskal-Wallis test were applied & & & \\
\hline Different alphabet within the different columns indicated significant differences $(\mathrm{p}<0.05)$ & \\
\hline
\end{tabular}


Table 4

Freshwater fish and seafood consumption (g/day/person) (median \pm IQR) by different gender in Peninsular Malaysia

\begin{tabular}{|c|c|c|c|c|c|}
\hline \multirow[t]{2}{*}{ Food category } & \multicolumn{3}{|c|}{ Gender } & \multirow{2}{*}{$\begin{array}{l}{ }_{p} \mathrm{p}- \\
\text { value }^{1}\end{array}$} & \multirow{2}{*}{$\begin{array}{l}{ }^{\#} \mathrm{p}- \\
\text { value }^{2}\end{array}$} \\
\hline & $\begin{array}{l}\text { Female } \\
(n= \\
1,859)\end{array}$ & $\begin{array}{l}\text { *Female (reproductive age) }(n= \\
1,091)\end{array}$ & $\begin{array}{l}\text { Male } \\
(n= \\
1,495)\end{array}$ & & \\
\hline Pelagic fish & $\begin{array}{l}70.7 \pm \\
73.3\end{array}$ & $40.0 \pm 60.7$ & $\begin{array}{l}58.7 \pm \\
49.7\end{array}$ & 0.234 & 0.057 \\
\hline Demersal fish & $\begin{array}{l}50.0 \pm \\
58.0\end{array}$ & $41.2 \pm 50.2$ & $\begin{array}{l}47.5 \pm \\
54.5\end{array}$ & 0.876 & 0.980 \\
\hline Total marine fish & $\begin{array}{l}58.7 \pm \\
91.0\end{array}$ & $44.0 \pm 76.8^{a}$ & $\begin{array}{l}53.7 \pm \\
75.7^{b}\end{array}$ & 0.215 & 0.034 \\
\hline $\begin{array}{l}\text { Total freshwater } \\
\text { fish }\end{array}$ & $\begin{array}{l}35.3 \pm \\
43.9\end{array}$ & $35.0 \pm 35.7$ & $\begin{array}{l}46.0 \pm \\
44.1\end{array}$ & 0.231 & 0.116 \\
\hline Cephalopods & $\begin{array}{l}43.3 \pm \\
31.7\end{array}$ & $31.9 \pm 34.1$ & $\begin{array}{l}64.5 \pm \\
40.3\end{array}$ & 0.231 & 0.329 \\
\hline Crustaceans & $\begin{array}{l}26.7 \pm \\
33.3\end{array}$ & $15.0 \pm 33.2$ & $\begin{array}{l}29.3 \pm \\
34.2\end{array}$ & 0.631 & 0.223 \\
\hline $\begin{array}{l}\text { Overall } \\
\text { consumption }\end{array}$ & $\begin{array}{l}86.0 \pm \\
96.3^{\mathrm{a}}\end{array}$ & $81.0 \pm 87.9^{b}$ & $\begin{array}{l}95.2 \pm \\
102^{\mathrm{ab}}\end{array}$ & 0.026 & 0.002 \\
\hline \multicolumn{6}{|c|}{ IQR - Inter-quartile Range; } \\
\hline \multicolumn{6}{|c|}{ * age between $15-49$ years old; } \\
\hline \multicolumn{6}{|c|}{ \#Kruskal-Wallis test were applied, } \\
\hline \multicolumn{6}{|c|}{$p$-value ${ }^{1}$ - differences between female and male, } \\
\hline \multicolumn{6}{|c|}{ p-value ${ }^{2}$ - differences between female at selected reproductive age and male; } \\
\hline
\end{tabular}

Fish and seafood consumption by three different major ethnics in Peninsular Malaysia were shown in Table 3. The overall fish and seafood consumption were led by the Malay ethnic ( $96.1 \pm 99.6 \mathrm{~g} / \mathrm{day})$ when compared to the Chinese $(66.0 \pm 88.0 \mathrm{~g} /$ day $)$ and Indians $(60.0 \pm 64.5 \mathrm{~g} /$ day $)$. The differences are significant at $p=0.000$. The Chinese significantly $(p=0.046)$ consumed lesser marine fish compared to the other two ethnic groups. In-contrary, the Indians consumed significantly $(p=0.009)$ the least pelagic fish compared to Malay ethnic and the Chinese. No significant differences $(p>0.05)$ were shown by different ethnicity towards consumption of demersal fish and freshwater fish, as well as cephalopods and crustaceans. Table 4 showed the consumption of different categories of fish and seafood by 
different gender. The overall results showed that fish and seafood consumption by male subjects ( $95.2 \pm$ $102 \mathrm{~g} /$ day $)$ were significantly $(p=0.026)$ higher when compared to the female $(86 \pm 96.3 \mathrm{~g} /$ day $)$ and female at reproductive age ( 15 to 49 years old) $(81 \pm 87.9 \mathrm{~g} /$ day). But no significant differences $(p>0.05)$ were shown for the other food categories between different genders except for total marine fish. Female at reproductive age consumed the least ( $44 \pm 76.85 \mathrm{~g} /$ day) of this fish categories compared to the other two groups. Table 5 showed consumption of fish and seafood by higher consumers. The consumption rates by the third quartile (75th percentile) consumers were 2.1 to 3.6 times greater than that the median consumers (50th percentile). The rates were even higher for the 90th and 95th percentile consumer groups at 2.9 to 4.4 times and 3.7 to 5.3 times, respectively.

Table 5

Freshwater fish and seafood consumption (g/day/person) *(median \pm IQR) by average and high consumers in Peninsular Malaysia

\begin{tabular}{|lllll|}
\hline Food category & \multicolumn{4}{c|}{ Higher consumer (percentiles) } \\
\cline { 2 - 5 } & $\mathbf{5 0}$ & $\mathbf{7 5}$ & $\mathbf{9 0}$ & $\mathbf{9 5}$ \\
\hline Pelagic fish & $58.3 \pm 63.8$ & $124.3 \pm 53.8$ & $171.0 \pm 63.8$ & $228.2 \pm 51.0$ \\
\hline Demersal fish & $43.7 \pm 48.7$ & $105.0 \pm 48.4$ & $138.0 \pm 92.0$ & $200.1 \pm 120.7$ \\
\hline Total marine fish & $51.1 \pm 77.3$ & $149.5 \pm 75.6$ & $199.0 \pm 71.7$ & $236.1 \pm 67.9$ \\
\hline Total freshwater fish & $36.7 \pm 45.7$ & $104.0 \pm 63.7$ & $151.7 \pm 58.6$ & $193.5 \pm 55.6$ \\
\hline Cephalopods & $45.0 \pm 35.2$ & $120.0 \pm 67.0$ & $148.3 \pm 59.3$ & $167.5 \pm 50.7$ \\
\hline Crustaceans & $22.9 \pm 30.2$ & $64.0 \pm 40.2$ & $100.5 \pm 50.0$ & $108.2 \pm 71.6$ \\
\hline Overall consumption & $89.0 \pm 100.7$ & $322.0 \pm 101.9$ & $380.5 \pm 117.2$ & $405.8 \pm 110.7$ \\
\hline IQR - Inter-quartile Range & & & \\
\hline
\end{tabular}

\section{Health Risk Assessment (Ewi, Mscw And Hq)}

Health risk assessment (EWI, MSCW and $\mathrm{HQ}$ ) of meHg from consumption of fish and seafood by populations in Peninsular Malaysia at different demographic factors were shown in Table 6 and the EWI were expressed in microgram per unit body weight per week ( $\mu \mathrm{g} / \mathrm{kg} \mathrm{BW} /$ week). The EWI estimated values showed results of below $1.6 \mu \mathrm{g} / \mathrm{kg}$ BW/week, the acceptable or tolerable levels recommended by the Joint FAO/WHO Expert Committee on Food Additives (JECFA) for all different demographic factors except for higher consumers at 75th percentile and above. The EWI for overall consumption of fish and seafood for higher consumers exceeded more than two times compared to the PTWI values and major sources of meHg intake were from the consumption of both the pelagic and demersal fish. The EWI value for overall consumption of fish and seafood consumed by the 50th percentile consumers were 0.6443 $\mu \mathrm{g} / \mathrm{kg} \mathrm{BW} /$ week (Table 6) and this value is corresponding to $40 \%$ of the PTWI. Major sources of meHg 
were from the consumption of demersal $(0.5149 \mu \mathrm{g} / \mathrm{kg} \mathrm{BW} /$ week) and pelagic $(0.4693 \mu \mathrm{g} / \mathrm{kg} \mathrm{BW} /$ week) fish at $32 \%$ and $29 \%$ of the PTWI value, respectively. Consumption of marine fish contributed to higher value of PTWI to all different demographic groups (the EWI range: $0.2988-0.6092 \mu \mathrm{g} / \mathrm{kg} \mathrm{BW} /$ week) (Table 6) except for the adolescents, where the major sources for this group is from the consumption of crustaceans $(0.3488 \mu \mathrm{g} / \mathrm{kg} \mathrm{BW} /$ week or $22 \%$ of PTWI) and cephalopods $(0.504 \mu \mathrm{g} / \mathrm{kg} \mathrm{BW} /$ week or $32 \%$ of PTWI). These contributed to the EWI values of $0.8196 \mu \mathrm{g} / \mathrm{kg} \mathrm{BW} /$ week or $51 \%$ of the PTWI for the overall consumption of fish and seafood among the adolescents. The value is at the highest rank when compared to the EWI values from the consumption of fish and seafood of the other groups. 
Table 6

Health risk assessment (EWI, MSCW and HQ) of meHg from consumption of fish/seafood by populations in Peninsular Malaysia at different demographic factors.

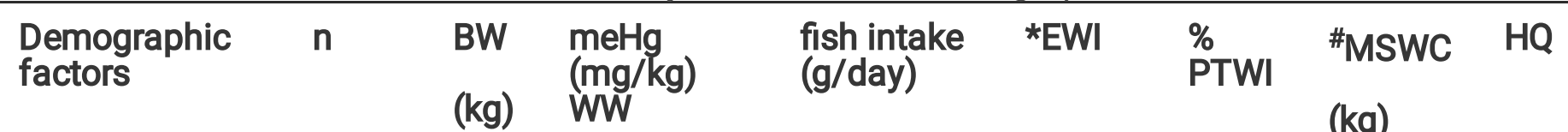

\section{Different age \\ groups}

Age $10-17 \quad 653$
years old

\begin{tabular}{|lcccccccc|}
\hline Pelagic fish & 135 & 45 & 0.069 & 22.0 & 0.2361 & 15 & 1.0435 & 0.3235 \\
\hline Demersal fish & 28 & 45 & 0.101 & 30.5 & 0.4792 & 30 & 0.7129 & 0.6564 \\
\hline $\begin{array}{l}\text { Total marine } \\
\text { fish }\end{array}$ & 156 & 45 & 0.074 & 26.0 & 0.2988 & 19 & 0.9745 & 0.4093 \\
\hline $\begin{array}{l}\text { Total } \\
\text { freshwater fish }\end{array}$ & 33 & 45 & 0.045 & 38.3 & 0.2681 & 17 & 1.6000 & 0.3673 \\
\hline Cephalopods & 43 & 45 & 0.041 & 80.0 & 0.5040 & 32 & 1.7778 & 0.6904 \\
\hline Crustaceans & 46 & 45 & 0.036 & 63.0 & 0.3488 & 22 & 2.0227 & 0.4779 \\
\hline $\begin{array}{l}\text { Overall } \\
\text { consumption }\end{array}$ & 505 & 45 & 0.062 & 84.9 & 0.8196 & 51 & 1.1602 & 1.1227 \\
\hline $\begin{array}{l}\text { Age 18-40 } \\
\text { years old }\end{array}$ & 1,209 & & & & & & & \\
\hline Pelagic fish & 546 & 60 & 0.069 & 44.0 & 0.3542 & 22 & 1.3913 & 0.4852 \\
\hline Demersal fish & 95 & 60 & 0.101 & 46.0 & 0.5420 & 34 & 0.9505 & 0.7425 \\
\hline $\begin{array}{l}\text { Total marine } \\
\text { fish }\end{array}$ & 581 & 60 & 0.074 & 50.0 & 0.4310 & 27 & 1.2993 & 0.5904 \\
\hline
\end{tabular}

BW - Body Weight (kg);

WW - Wet Weight;

*EWI - Estimated Weekly Intake ( $\mu \mathrm{g} / \mathrm{kg}$ BW/week);

**age between $15-49$ years old;

Provisional Tolerable Weekly Intake (PTWI) for $\mathrm{MeHg}=1.6 \mu \mathrm{g} / \mathrm{kg} \mathrm{BW} /$ week (WHO 2011).

MSWC - Maximum Safe Weekly Consumption (kg);

NC- MSCW for higher consumer (75th, 90th, 95th centile) was not calculated as the EWI values for all fish and seafood groups were either nearly or above the PTWl;

HQ - Hazard Quotient 


\begin{tabular}{|c|c|c|c|c|c|c|c|c|}
\hline $\begin{array}{l}\text { Demographic } \\
\text { factors }\end{array}$ & $\mathrm{n}$ & $\begin{array}{l}\text { BW } \\
(\mathrm{kg})\end{array}$ & $\begin{array}{l}\mathrm{meHg} \\
(\mathrm{mg} / \mathrm{kg}) \\
\mathrm{WW}\end{array}$ & $\begin{array}{l}\text { fish intake } \\
\text { (g/day) }\end{array}$ & *EWI & $\begin{array}{l}\% \\
\text { PTWI }\end{array}$ & $\begin{array}{l}\text { \#MSWC } \\
(\mathrm{kg})\end{array}$ & $\mathrm{HQ}$ \\
\hline $\begin{array}{l}\text { Total } \\
\text { freshwater fish }\end{array}$ & 53 & 60 & 0.045 & 35.3 & 0.1853 & 12 & 2.1333 & 0.2539 \\
\hline Cephalopods & 71 & 60 & 0.041 & 30.0 & 0.1418 & 9 & 2.3704 & 0.1942 \\
\hline Crustaceans & 104 & 60 & 0.036 & 13.3 & 0.0552 & 3 & 2.6970 & 0.0757 \\
\hline $\begin{array}{l}\text { Overall } \\
\text { consumption }\end{array}$ & 894 & 60 & 0.062 & 82.0 & 0.5937 & 37 & 1.5470 & 0.8132 \\
\hline $\begin{array}{l}\text { Age 41-60 } \\
\text { years old }\end{array}$ & 1,073 & & & & & & & \\
\hline Pelagic fish & 562 & 65 & 0.069 & 48.7 & 0.3619 & 23 & 1.5072 & 0.4957 \\
\hline Demersal fish & 118 & 65 & 0.101 & 35.0 & 0.3807 & 24 & 1.0297 & 0.5215 \\
\hline $\begin{array}{l}\text { Total marine } \\
\text { fish }\end{array}$ & 602 & 65 & 0.074 & 60.0 & 0.4774 & 30 & 1.4076 & 0.6540 \\
\hline $\begin{array}{l}\text { Total } \\
\text { freshwater fish }\end{array}$ & 61 & 65 & 0.045 & 36.7 & 0.1779 & 11 & 2.3111 & 0.2436 \\
\hline Cephalopods & 80 & 65 & 0.041 & 40.0 & 0.1745 & 11 & 2.5679 & 0.2390 \\
\hline Crustaceans & 93 & 65 & 0.036 & 21.0 & 0.0805 & 5 & 2.9217 & 0.1103 \\
\hline $\begin{array}{l}\text { Overall } \\
\text { consumption }\end{array}$ & 193 & 65 & 0.062 & 95.8 & 0.6402 & 40 & 1.6759 & 0.8770 \\
\hline $\begin{array}{l}\text { Age } \geq 60 \text { years } \\
\text { old }\end{array}$ & 422 & & & & & & & \\
\hline Pelagic fish & 209 & 59 & 0.069 & 48.3 & 0.3954 & 25 & 1.3681 & 0.5417 \\
\hline Demersal fish & 60 & 59 & 0.101 & 52.0 & 0.6231 & 39 & 0.9347 & 0.8536 \\
\hline \multicolumn{9}{|c|}{ BW - Body Weight (kg); } \\
\hline \multicolumn{9}{|c|}{ WW - Wet Weight; } \\
\hline \multicolumn{9}{|c|}{ *EWI - Estimated Weekly Intake ( $\mu \mathrm{g} / \mathrm{kg} \mathrm{BW/ \text {week});}$} \\
\hline \multicolumn{9}{|c|}{ **age between $15-49$ years old; } \\
\hline \multicolumn{9}{|c|}{ Provisional Tolerable Weekly Intake (PTWI) for MeHg = $1.6 \mu \mathrm{g} / \mathrm{kg}$ BW/week (WHO 2011). } \\
\hline \multicolumn{9}{|c|}{ MSWC - Maximum Safe Weekly Consumption (kg); } \\
\hline \multicolumn{9}{|c|}{$\begin{array}{l}\text { NC- MSCW for higher consumer (75th, 90th, 95th centile) was not calculated as the EWI values for all } \\
\text { fish and seafood groups were either nearly or above the PTWl; }\end{array}$} \\
\hline HQ - Hazard Quc & & & & & & & & \\
\hline
\end{tabular}




\begin{tabular}{|c|c|c|c|c|c|c|c|c|}
\hline $\begin{array}{l}\text { Demographic } \\
\text { factors }\end{array}$ & $\mathrm{n}$ & $\begin{array}{l}\text { BW } \\
(\mathrm{kg})\end{array}$ & $\begin{array}{l}\mathrm{meHg} \\
(\mathrm{mg} / \mathrm{kg}) \\
\mathrm{ww}\end{array}$ & $\begin{array}{l}\text { fish intake } \\
\text { (g/day) }\end{array}$ & *EWI & $\begin{array}{l}\% \\
\text { PTWI }\end{array}$ & $\begin{array}{l}\text { \#MSWC } \\
(\mathrm{kg})\end{array}$ & HQ \\
\hline $\begin{array}{l}\text { Total marine } \\
\text { fish }\end{array}$ & 223 & 59 & 0.074 & 66.0 & 0.5786 & 36 & 1.2777 & 0.7925 \\
\hline $\begin{array}{l}\text { Total } \\
\text { freshwater fish }\end{array}$ & 15 & 59 & 0.045 & 36.7 & 0.1959 & 12 & 2.0978 & 0.2684 \\
\hline Cephalopods & 18 & 59 & 0.041 & 29.8 & 0.1432 & 9 & 2.3309 & 0.1962 \\
\hline Crustaceans & 27 & 59 & 0.036 & 13.3 & 0.0562 & 4 & 2.6520 & 0.0769 \\
\hline $\begin{array}{l}\text { Overall } \\
\text { consumption }\end{array}$ & 340 & 59 & 0.062 & 104.0 & 0.7657 & 48 & 1.5212 & 1.0489 \\
\hline \multicolumn{9}{|l|}{$\begin{array}{l}\text { Table 6: } \\
\text { continue }\end{array}$} \\
\hline $\begin{array}{l}\text { Demographic } \\
\text { factors }\end{array}$ & $n$ & $\begin{array}{l}\text { BW } \\
(\mathrm{kg})\end{array}$ & $\begin{array}{l}\mathrm{meHg} \\
(\mathrm{mg} / \mathrm{kg} \\
\mathrm{WW})\end{array}$ & $\begin{array}{l}\text { fish intake } \\
\text { (g/day) }\end{array}$ & *EWI & $\begin{array}{l}\% \\
\text { PTWI }\end{array}$ & $\begin{array}{l}{ }^{\#} \text { MSWC } \\
(\mathrm{kg})\end{array}$ & $\mathrm{HQ}$ \\
\hline \multicolumn{9}{|l|}{$\begin{array}{l}\text { Different } \\
\text { ethnicity }\end{array}$} \\
\hline Malays & 1,495 & & & & & & & \\
\hline Pelagic fish & 1,219 & 59 & 0.069 & 58.7 & 0.4805 & 30 & 1.3681 & 0.6583 \\
\hline Demersal fish & 264 & 59 & 0.101 & 46.0 & 0.5512 & 34 & 0.9347 & 0.7551 \\
\hline $\begin{array}{l}\text { Total marine } \\
\text { fish }\end{array}$ & 1,314 & 59 & 0.074 & 69.5 & 0.6092 & 38 & 1.2777 & 0.8346 \\
\hline $\begin{array}{l}\text { Total } \\
\text { freshwater fish }\end{array}$ & 150 & 59 & 0.045 & 36.7 & 0.1959 & 12 & 2.0978 & 0.2684 \\
\hline Cephalopods & 196 & 59 & 0.041 & 47.8 & 0.2297 & 14 & 2.3309 & 0.3146 \\
\hline \multicolumn{9}{|c|}{ BW - Body Weight (kg); } \\
\hline \multicolumn{9}{|c|}{ WW - Wet Weight; } \\
\hline \multicolumn{9}{|c|}{ *EWI - Estimated Weekly Intake ( $\mu \mathrm{g} / \mathrm{kg} \mathrm{BW/ \text {week});}$} \\
\hline \multicolumn{9}{|c|}{ **age between $15-49$ years old; } \\
\hline \multicolumn{9}{|c|}{ Provisional Tolerable Weekly Intake (PTWI) for MeHg = $1.6 \mu \mathrm{g} / \mathrm{kg} \mathrm{BW/week} \mathrm{(WHO} \mathrm{2011).}$} \\
\hline \multicolumn{9}{|c|}{ MSWC - Maximum Safe Weekly Consumption (kg); } \\
\hline \multicolumn{9}{|c|}{$\begin{array}{l}\text { NC- MSCW for higher consumer (75th, 90th, 95th centile) was not calculated as the EWI values for all } \\
\text { fish and seafood groups were either nearly or above the PTWI; }\end{array}$} \\
\hline HQ - Hazard Qu & & & & & & & & \\
\hline
\end{tabular}




\begin{tabular}{|c|c|c|c|c|c|c|c|c|}
\hline $\begin{array}{l}\text { Demographic } \\
\text { factors }\end{array}$ & $\mathrm{n}$ & $\begin{array}{l}\text { BW } \\
(\mathrm{kg})\end{array}$ & $\begin{array}{l}\mathrm{meHg} \\
(\mathrm{mg} / \mathrm{kg}) \\
\mathrm{WW}\end{array}$ & $\begin{array}{l}\text { fish intake } \\
\text { (g/day) }\end{array}$ & *EWI & $\begin{array}{l}\% \\
\text { PTWI }\end{array}$ & $\begin{array}{l}\text { \#MSWC } \\
(\mathrm{kg})\end{array}$ & HQ \\
\hline Crustaceans & 219 & 59 & 0.036 & 21.3 & 0.0900 & 6 & 2.6520 & 0.1232 \\
\hline $\begin{array}{l}\text { Overall } \\
\text { consumption }\end{array}$ & 2,116 & 59 & 0.062 & 96.1 & 0.7075 & 44 & 1.5212 & 0.9692 \\
\hline Chinese & 457 & & & & & & & \\
\hline Pelagic fish & 125 & 60 & 0.069 & 55.3 & 0.4452 & 28 & 1.3913 & 0.6098 \\
\hline Demersal fish & 21 & 60 & 0.101 & 37.3 & 0.4395 & 27 & 0.9505 & 0.6021 \\
\hline $\begin{array}{l}\text { Total marine } \\
\text { fish }\end{array}$ & 131 & 60 & 0.074 & 45.0 & 0.3879 & 24 & 1.2993 & 0.5314 \\
\hline $\begin{array}{l}\text { Total } \\
\text { freshwater fish }\end{array}$ & 5 & 60 & 0.045 & 42.7 & 0.2242 & 14 & 2.1333 & 0.3071 \\
\hline Cephalopods & 10 & 60 & 0.041 & 53.3 & 0.2518 & 16 & 2.3704 & 0.3450 \\
\hline Crustaceans & 15 & 60 & 0.036 & 3.8 & 0.0158 & 1 & 2.6970 & 0.0216 \\
\hline $\begin{array}{l}\text { Overall } \\
\text { consumption }\end{array}$ & 274 & 60 & 0.062 & 66.0 & 0.4778 & 30 & 1.5470 & 0.6546 \\
\hline Indians & 270 & & & & & & & \\
\hline Pelagic fish & 89 & 55 & 0.069 & 20.0 & 0.1756 & 11 & 1.2754 & 0.2406 \\
\hline Demersal fish & 10 & 55 & 0.101 & 42.3 & 0.5437 & 34 & 0.8713 & 0.7449 \\
\hline $\begin{array}{l}\text { Total marine } \\
\text { fish }\end{array}$ & 95 & 55 & 0.074 & 73.3 & 0.6893 & 43 & 1.1911 & 0.9442 \\
\hline $\begin{array}{l}\text { Total } \\
\text { freshwater fish }\end{array}$ & 1 & 55 & 0.045 & 44.0 & 0.2520 & 16 & 1.9556 & 0.3452 \\
\hline
\end{tabular}

BW - Body Weight $(\mathrm{kg})$;

WW - Wet Weight;

*EWI - Estimated Weekly Intake ( $\mu \mathrm{g} / \mathrm{kg}$ BW/week);

**age between $15-49$ years old;

Provisional Tolerable Weekly Intake (PTWI) for MeHg = $1.6 \mu \mathrm{g} / \mathrm{kg}$ BW/week (WHO 2011).

MSWC - Maximum Safe Weekly Consumption (kg);

NC- MSCW for higher consumer (75th, 90th, 95th centile) was not calculated as the EWI values for all fish and seafood groups were either nearly or above the PTWl;

HQ - Hazard Quotient 


\begin{tabular}{|c|c|c|c|c|c|c|c|c|}
\hline $\begin{array}{l}\text { Demographic } \\
\text { factors }\end{array}$ & $\mathrm{n}$ & $\begin{array}{l}\text { BW } \\
(\mathrm{kg})\end{array}$ & $\begin{array}{l}\mathrm{meHg} \\
(\mathrm{mg} / \mathrm{kg}) \\
\mathrm{WW}\end{array}$ & $\begin{array}{l}\text { fish intake } \\
\text { (g/day) }\end{array}$ & *EWI & $\begin{array}{l}\% \\
\text { PTWI }\end{array}$ & $\begin{array}{l}\text { \#MSWC } \\
(\mathrm{kg})\end{array}$ & HQ \\
\hline Cephalopods & 6 & 55 & 0.041 & 26.7 & 0.1376 & 9 & 2.1728 & 0.1885 \\
\hline Crustaceans & 36 & 55 & 0.036 & 70.0 & 0.3171 & 20 & 2.4722 & 0.4344 \\
\hline $\begin{array}{l}\text { Overall } \\
\text { consumption }\end{array}$ & 196 & 55 & 0.062 & 60.0 & 0.4739 & 30 & 1.4181 & 0.6492 \\
\hline \multicolumn{9}{|l|}{$\begin{array}{l}\text { Different } \\
\text { gender }\end{array}$} \\
\hline Male & 1,495 & & & & & & & \\
\hline Pelagic fish & 645 & 62 & 0.069 & 58.7 & 0.4573 & 29 & 1.4377 & 0.6264 \\
\hline Demersal fish & 130 & 62 & 0.101 & 47.5 & 0.5417 & 34 & 0.9822 & 0.7420 \\
\hline $\begin{array}{l}\text { Total marine } \\
\text { fish }\end{array}$ & 690 & 62 & 0.074 & 53.7 & 0.4480 & 28 & 1.3426 & 0.6136 \\
\hline $\begin{array}{l}\text { Total } \\
\text { freshwater fish }\end{array}$ & 83 & 62 & 0.045 & 46.0 & 0.2337 & 15 & 2.2044 & 0.3202 \\
\hline Cephalopods & 97 & 62 & 0.041 & 53.3 & 0.2437 & 15 & 2.4494 & 0.3339 \\
\hline Crustaceans & 112 & 62 & 0.036 & 28.0 & 0.1125 & 7 & 2.7869 & 0.1541 \\
\hline $\begin{array}{l}\text { Overall } \\
\text { consumption }\end{array}$ & 1,142 & 62 & 0.062 & 95.2 & 0.6670 & 42 & 1.5986 & 0.9137 \\
\hline \multicolumn{9}{|l|}{$\begin{array}{l}\text { Table 6: } \\
\text { continue }\end{array}$} \\
\hline $\begin{array}{l}\text { Demographic } \\
\text { factors }\end{array}$ & $n$ & $\begin{array}{l}\text { BW } \\
(\mathrm{kg})\end{array}$ & $\begin{array}{l}\mathrm{meHg} \\
(\mathrm{mg} / \mathrm{kg} \\
\mathrm{WW})\end{array}$ & $\begin{array}{l}\text { fish intake } \\
\text { (g/day) }\end{array}$ & *EWI & $\begin{array}{l}\% \\
\text { PTWI }\end{array}$ & $\begin{array}{l}\text { \#MSWC } \\
(\mathrm{kg})\end{array}$ & HQ \\
\hline
\end{tabular}

BW - Body Weight $(\mathrm{kg})$;

WW - Wet Weight;

*EWI - Estimated Weekly Intake ( $\mu \mathrm{g} / \mathrm{kg}$ BW/week);

**age between $15-49$ years old;

Provisional Tolerable Weekly Intake (PTWI) for MeHg = $1.6 \mu \mathrm{g} / \mathrm{kg}$ BW/week (WHO 2011).

MSWC - Maximum Safe Weekly Consumption (kg);

NC- MSCW for higher consumer (75th, 90th, 95th centile) was not calculated as the EWI values for all fish and seafood groups were either nearly or above the PTWl;

HQ - Hazard Quotient 


\begin{tabular}{|c|c|c|c|c|c|c|c|c|}
\hline $\begin{array}{l}\text { Demographic } \\
\text { factors }\end{array}$ & $\mathrm{n}$ & $\begin{array}{l}\text { BW } \\
(\mathrm{kg})\end{array}$ & $\begin{array}{l}\mathrm{meHg} \\
(\mathrm{mg} / \mathrm{kg}) \\
\mathrm{Ww}\end{array}$ & $\begin{array}{l}\text { fish intake } \\
\text { (g/day) }\end{array}$ & *EWI & $\begin{array}{l}\% \\
\text { PTWI }\end{array}$ & $\begin{array}{l}\text { \#MSWC } \\
(\mathrm{kg})\end{array}$ & HQ \\
\hline Female & 1,859 & & & & & & & \\
\hline Pelagic fish & 806 & 57 & 0.069 & 70.7 & 0.5991 & 37 & 1.3217 & 0.8207 \\
\hline Demersal fish & 171 & 57 & 0.101 & 50.0 & 0.6202 & 39 & 0.9030 & 0.8496 \\
\hline $\begin{array}{l}\text { Total marine } \\
\text { fish }\end{array}$ & 871 & 57 & 0.074 & 58.7 & 0.5326 & 33 & 1.2344 & 0.7296 \\
\hline $\begin{array}{l}\text { Total } \\
\text { freshwater fish }\end{array}$ & 79 & 57 & 0.045 & 35.3 & 0.1951 & 12 & 2.0267 & 0.2672 \\
\hline Cephalopods & 115 & 57 & 0.041 & 43.3 & 0.2154 & 13 & 2.2519 & 0.2950 \\
\hline Crustaceans & 158 & 57 & 0.036 & 26.7 & 0.1167 & 7 & 2.5621 & 0.1599 \\
\hline $\begin{array}{l}\text { Overall } \\
\text { consumption }\end{array}$ & 1,474 & 57 & 0.062 & 86.0 & 0.6554 & 41 & 1.4696 & 0.8978 \\
\hline $\begin{array}{l}\text { Female } \\
\text { **(reproductive } \\
\text { age) }\end{array}$ & 1,091 & & & & & & & \\
\hline Pelagic fish & 477 & 59 & 0.069 & 40.0 & 0.3275 & 20 & 1.3681 & 0.4486 \\
\hline Demersal fish & 86 & 59 & 0.101 & 41.2 & 0.4937 & 31 & 0.9347 & 0.6763 \\
\hline $\begin{array}{l}\text { Total marine } \\
\text { fish }\end{array}$ & 514 & 59 & 0.074 & 44.0 & 0.3857 & 24 & 1.2777 & 0.5284 \\
\hline $\begin{array}{l}\text { Total } \\
\text { freshwater fish }\end{array}$ & 49 & 59 & 0.045 & 35.0 & 0.1869 & 12 & 2.0978 & 0.2560 \\
\hline Cephalopods & 74 & 59 & 0.041 & 31.9 & 0.1533 & 10 & 2.3309 & 0.2100 \\
\hline
\end{tabular}

BW - Body Weight $(\mathrm{kg})$;

WW - Wet Weight;

*EWI - Estimated Weekly Intake ( $\mu \mathrm{g} / \mathrm{kg}$ BW/week);

**age between $15-49$ years old;

Provisional Tolerable Weekly Intake (PTWI) for MeHg = $1.6 \mu \mathrm{g} / \mathrm{kg}$ BW/week (WHO 2011).

MSWC - Maximum Safe Weekly Consumption (kg);

NC- MSCW for higher consumer (75th, 90th, 95th centile) was not calculated as the EWI values for all fish and seafood groups were either nearly or above the PTWl;

HQ - Hazard Quotient 


\begin{tabular}{|c|c|c|c|c|c|c|c|c|}
\hline $\begin{array}{l}\text { Demographic } \\
\text { factors }\end{array}$ & $\mathbf{n}$ & $\begin{array}{l}\text { BW } \\
(\mathrm{kg})\end{array}$ & $\begin{array}{l}\mathrm{meHg} \\
(\mathrm{mg} / \mathrm{kg}) \\
\mathrm{ww}\end{array}$ & $\begin{array}{l}\text { fish intake } \\
\text { (g/day) }\end{array}$ & *EWI & $\begin{array}{l}\% \\
\text { PTWI }\end{array}$ & $\begin{array}{l}\text { \#MSWC } \\
(\mathrm{kg})\end{array}$ & $\mathrm{HQ}$ \\
\hline Crustaceans & 96 & 59 & 0.036 & 15.0 & 0.0633 & 4 & 2.6520 & 0.0868 \\
\hline $\begin{array}{l}\text { Overall } \\
\text { consumption }\end{array}$ & 841 & 59 & 0.062 & 81.0 & 0.5964 & 37 & 1.5212 & 0.8169 \\
\hline \multicolumn{9}{|c|}{ Consumption rate by percentile } \\
\hline $\begin{array}{l}\text { Median (50 } \\
\text { percentile) }\end{array}$ & 3,357 & & & & & & & \\
\hline Pelagic fish & 1452 & 60 & 0.069 & 58.3 & 0.4693 & 29 & 1.3913 & 0.6429 \\
\hline Demersal fish & 301 & 60 & 0.101 & 43.7 & 0.5149 & 32 & 0.9505 & 0.7054 \\
\hline $\begin{array}{l}\text { Total marine } \\
\text { fish }\end{array}$ & 1562 & 60 & 0.074 & 51.1 & 0.4405 & 28 & 1.2993 & 0.6034 \\
\hline $\begin{array}{l}\text { Total } \\
\text { freshwater fish }\end{array}$ & 162 & 60 & 0.045 & 36.7 & 0.1927 & 12 & 2.1333 & 0.2639 \\
\hline Cephalopods & 212 & 60 & 0.041 & 45.0 & 0.2126 & 13 & 2.3704 & 0.2913 \\
\hline Crustaceans & 270 & 60 & 0.036 & 22.9 & 0.0951 & 6 & 2.6970 & 0.1303 \\
\hline $\begin{array}{l}\text { Overall } \\
\text { consumption }\end{array}$ & 2,619 & 60 & 0.062 & 89.0 & 0.6443 & 40 & 1.5470 & 0.8827 \\
\hline \multicolumn{9}{|l|}{ High consumer } \\
\hline $\begin{array}{l}\text { (75th } \\
\text { percentile) }\end{array}$ & 657 & & & & & & & \\
\hline Pelagic fish & 323 & 60 & 0.069 & 124.3 & 1.0006 & 63 & NC & 1.3707 \\
\hline Demersal fish & 72 & 60 & 0.101 & 105.0 & 1.2373 & 77 & $\mathrm{NC}$ & 1.6949 \\
\hline
\end{tabular}

BW - Body Weight $(\mathrm{kg})$;

WW - Wet Weight;

*EWI - Estimated Weekly Intake ( $\mu \mathrm{g} / \mathrm{kg}$ BW/week);

**age between $15-49$ years old;

Provisional Tolerable Weekly Intake (PTWI) for MeHg = $1.6 \mu \mathrm{g} / \mathrm{kg}$ BW/week (WHO 2011).

MSWC - Maximum Safe Weekly Consumption (kg);

NC- MSCW for higher consumer (75th, 90th, 95th centile) was not calculated as the EWI values for all fish and seafood groups were either nearly or above the PTWl;

HQ - Hazard Quotient 


\begin{tabular}{|c|c|c|c|c|c|c|c|c|}
\hline $\begin{array}{l}\text { Demographic } \\
\text { factors }\end{array}$ & $n$ & $\begin{array}{l}\text { BW } \\
(\mathrm{kg})\end{array}$ & $\begin{array}{l}\mathrm{meHg} \\
(\mathrm{mg} / \mathrm{kg}) \\
\mathrm{wW}\end{array}$ & $\begin{array}{l}\text { fish intake } \\
\text { (g/day) }\end{array}$ & *EWI & $\begin{array}{l}\% \\
\text { PTWI }\end{array}$ & $\begin{array}{l}\text { \#MSWC } \\
(\mathrm{kg})\end{array}$ & HQ \\
\hline $\begin{array}{l}\text { Total marine } \\
\text { fish }\end{array}$ & 368 & 60 & 0.074 & 149.5 & 1.2887 & 81 & NC & 1.7653 \\
\hline $\begin{array}{l}\text { Total } \\
\text { freshwater fish }\end{array}$ & 36 & 60 & 0.045 & 104.0 & 0.5460 & 34 & NC & 0.7479 \\
\hline Cephalopods & 57 & 60 & 0.041 & 120.0 & 0.5670 & 35 & NC & 0.7767 \\
\hline Crustaceans & 73 & 60 & 0.036 & 64.0 & 0.2658 & 17 & NC & 0.3641 \\
\hline $\begin{array}{l}\text { Overall } \\
\text { consumption }\end{array}$ & 148 & 60 & 0.062 & 322.0 & 2.3312 & 146 & NC & 3.1935 \\
\hline \multicolumn{9}{|l|}{$\begin{array}{l}\text { Table 6: } \\
\text { continue }\end{array}$} \\
\hline $\begin{array}{l}\text { Demographic } \\
\text { factors }\end{array}$ & $n$ & $\begin{array}{l}\text { BW } \\
(\mathrm{kg})\end{array}$ & $\begin{array}{l}\mathrm{meHg} \\
(\mathrm{mg} / \mathrm{kg} \\
\mathrm{WW})\end{array}$ & $\begin{array}{l}\text { fish intake } \\
\text { (g/day) }\end{array}$ & *EWI & $\begin{array}{l}\% \\
\text { PTWI }\end{array}$ & $\begin{array}{l}{ }^{\#} \text { MSWC } \\
(\mathrm{kg})\end{array}$ & $\mathrm{HQ}$ \\
\hline $\begin{array}{l}\text { (90th } \\
\text { percentile) }\end{array}$ & 264 & & & & & & & \\
\hline Pelagic fish & 117 & 60 & 0.069 & 171.0 & 1.3766 & 86 & NC & 1.8857 \\
\hline Demersal fish & 33 & 60 & 0.101 & 138.0 & 1.6261 & 102 & NC & 2.2275 \\
\hline $\begin{array}{l}\text { Total marine } \\
\text { fish }\end{array}$ & 181 & 60 & 0.074 & 199.0 & 1.7153 & 107 & NC & 2.3498 \\
\hline $\begin{array}{l}\text { Total } \\
\text { freshwater fish }\end{array}$ & 13 & 60 & 0.045 & 151.7 & 0.7964 & 50 & NC & 1.0910 \\
\hline Cephalopods & 39 & 60 & 0.041 & 148.3 & 0.7007 & 44 & NC & 0.9599 \\
\hline Crustaceans & 34 & 60 & 0.036 & 100.5 & 0.4174 & 26 & NC & 0.5717 \\
\hline \multicolumn{9}{|c|}{ BW - Body Weight (kg); } \\
\hline \multicolumn{9}{|c|}{ WW - Wet Weight; } \\
\hline \multicolumn{9}{|c|}{ *EWI - Estimated Weekly Intake ( $\mu \mathrm{g} / \mathrm{kg} \mathrm{BW/ \text {week});}$} \\
\hline \multicolumn{9}{|c|}{ **age between $15-49$ years old; } \\
\hline \multicolumn{9}{|c|}{ Provisional Tolerable Weekly Intake (PTWI) for MeHg = $1.6 \mu \mathrm{g} / \mathrm{kg} \mathrm{BW/week} \mathrm{(WHO} \mathrm{2011).}$} \\
\hline \multicolumn{9}{|c|}{ MSWC - Maximum Safe Weekly Consumption (kg); } \\
\hline \multicolumn{9}{|c|}{$\begin{array}{l}\text { NC- MSCW for higher consumer (75th, 90th, } 95 \text { th centile) was not calculated as the EWI values for all } \\
\text { fish and seafood groups were either nearly or above the PTWl; }\end{array}$} \\
\hline HQ - Hazard Qu & & & & & & & & \\
\hline
\end{tabular}




\begin{tabular}{|c|c|c|c|c|c|c|c|c|}
\hline $\begin{array}{l}\text { Demographic } \\
\text { factors }\end{array}$ & $\mathrm{n}$ & $\begin{array}{l}\text { BW } \\
(\mathrm{kg})\end{array}$ & $\begin{array}{l}\mathrm{meHg} \\
(\mathrm{mg} / \mathrm{kg}) \\
\mathrm{WW}\end{array}$ & $\begin{array}{l}\text { fish intake } \\
\text { (g/day) }\end{array}$ & *EWI & $\begin{array}{l}\% \\
\text { PTWI }\end{array}$ & $\begin{array}{l}\text { \#MSWC } \\
(\mathrm{kg})\end{array}$ & HQ \\
\hline $\begin{array}{l}\text { Overall } \\
\text { consumption }\end{array}$ & 81 & 60 & 0.062 & 380.5 & 2.7548 & 172 & NC & 3.7737 \\
\hline $\begin{array}{l}\text { (95th } \\
\text { percentile) }\end{array}$ & 131 & & & & & & & \\
\hline Pelagic fish & 47 & 60 & 0.069 & 228.2 & 1.8370 & 115 & NC & 2.5165 \\
\hline Demersal fish & 17 & 60 & 0.101 & 200.1 & 2.3578 & 147 & NC & 3.2299 \\
\hline $\begin{array}{l}\text { Total marine } \\
\text { fish }\end{array}$ & 109 & 60 & 0.074 & 236.1 & 2.0351 & 127 & NC & 2.7879 \\
\hline $\begin{array}{l}\text { Total } \\
\text { freshwater fish }\end{array}$ & 6 & 60 & 0.045 & 193.5 & 1.0159 & 63 & NC & 1.3916 \\
\hline Cephalopods & 22 & 60 & 0.041 & 167.5 & 0.7914 & 49 & NC & 1.0842 \\
\hline Crustaceans & 28 & 60 & 0.036 & 108.2 & 0.4493 & 28 & NC & 0.6155 \\
\hline $\begin{array}{l}\text { Overall } \\
\text { consumption }\end{array}$ & 58 & 60 & 0.062 & 405.8 & 2.9379 & 184 & NC & 4.0246 \\
\hline \multicolumn{9}{|c|}{ BW - Body Weight (kg); } \\
\hline \multicolumn{9}{|c|}{ WW - Wet Weight; } \\
\hline \multicolumn{9}{|c|}{ *EWI - Estimated Weekly Intake ( $\mu \mathrm{g} / \mathrm{kg} \mathrm{BW/week);}$} \\
\hline \multicolumn{9}{|c|}{ **age between $15-49$ years old; } \\
\hline \multicolumn{9}{|c|}{ Provisional Tolerable Weekly Intake (PTWI) for MeHg = $1.6 \mu \mathrm{g} / \mathrm{kg} \mathrm{BW} /$ week (WHO 2011). } \\
\hline \multicolumn{9}{|c|}{ MSWC - Maximum Safe Weekly Consumption (kg); } \\
\hline \multicolumn{9}{|c|}{$\begin{array}{l}\text { NC- MSCW for higher consumer ( } 75 \text { th, } 90 \text { th, } 95 \text { th centile) was not calculated as the EWI values for all } \\
\text { fish and seafood groups were either nearly or above the PTWl; }\end{array}$} \\
\hline HQ - Hazard Qu & & & & & & & & \\
\hline
\end{tabular}

MSWC values in $\mathrm{kg}$ are given on fish and seafood by group basis (Table 6). The amounts of demersal fish from Peninsular Malaysia which should be consumed by all population groups to reach the PTWI for meHg would be below $1 \mathrm{~kg} /$ week and very much lower $(<713 \mathrm{~g} /$ week) for the adolescents. The adolescents were allowed to consumed $<2 \mathrm{~kg}$ per week (range: $1.04-2.02 \mathrm{~kg} /$ week) for all types of fish and seafood. To be more specific, about a kilogram for pelagic fish, one and a half kilogram to two kilograms of fresh water fish, the cephalopods and crustaceans. While for the adults, they were allowed to consume greater amount $(<2.5 \mathrm{~kg} /$ week) of marine and freshwater fish per week.

Table 6 also showed a summary of the HQ values for meHg from the consumption of fish and seafood by population in Peninsular Malaysia at different demographic background. The HQ is an integrated risk 
index that compares the ingested amount of meHg with the standard reference dose. Results from this study revealed that the overall consumption of fish and seafood by the adolescents and the elderly exceeded one. This indicating the non-acceptable level of non-carcinogenic adverse health effects. Still, the $\mathrm{HQ}<1$ for the other groups of population at different types of fish and seafood categories assumes that daily exposure is not likely to cause negative health effects during the lifetime of the Malaysian population. High $\mathrm{HQ}$ values were contributed from the consumption of the demersal fish which exceeded range between 0.5 to 0.8 or nearly $90 \%$ of the $\mathrm{HQ}$ values. For the adolescents, high $\mathrm{HQ}$ values were also contributed from the consumption of demersal fish but the highest is from consumption of cephalopods. The high consumer at 75th percentile and above, the HQ values reached of up to 4.3 which indicated presence of adverse effects due to meHg intoxication.

\section{Discussion}

This study was carried out to assess the potential health risk of meHg exposure to population of Peninsular Malaysia from consumption of freshwater fish and seafood. We used two approaches for the estimation of human health risks to meHg in fish and seafood; the widely applied is the comparison with the PTWIs which representing the amount of meHg that can be ingested over a lifetime without appreciative risks (WHO 2011). Another approach is to determine risk through the value of HQ (USEPA 2000). This HQ value is an integrated index that compares the ingested amount of meHg with the standard reference dose where $\mathrm{HQ}<1$ signifies that the level of exposure is lower than the reference dose. It assumes a daily exposure at this level is not likely to cause any negatives health effects during a lifetime in a human population.

It is well documented that meHg occurs at high percentage in fish muscle and is the most toxic form of $\mathrm{Hg}$. We analyzed THg in freshwater fish and seafood, these data were used to estimate meHg intakes for risk assessment data. In our earlier published data (Ahmad et al $2015^{\mathrm{ab}}$ ), we presented and discussed in detail on THg levels in 46 species of commonly consumed marine fish samples and other seafood as well (cephalopods; 8 species and crustaceans; 12 species). The relationship between $\mathrm{THg}$ levels and size of samples (length and weight) was also discussed, and THg burden sampled from fish and seafood at different habitats, family group, and areas were compared. Previous results revealed only $1 \%$ or three samples of demersal fish (bluespotted singray (Neotrygon kuhlii), honeycomb stingray (Himantura uarnak), John's snapper (Lutjanus ruselli)) had very high levels of THg (Ahmad et al 2015ª). However, only one samples exceeded the Malaysian and international guidelines (FAO/WHO 2006; Malaysian Food Regulation 1985) when considering $95 \%$ or more of THg in the edible portion of seafood in the form of meHg (Khaniki et al 2005). The previous results for THg levels in crustaceans and cephalopods were either similar or reasonably low when compared to values published in various locations throughout the world (Ahmad et al 2015b), and none of these samples surpassed the guidelines. As data for meHg analysis in seafood for this country is scanty, we used levels recently reported by Annual and co-workers (2018) for the nearest estimation, yet, only one sample each was analyses for crustacean (Metapenaeus affinis) and cephalopod (Loligo duvauceli). The re-calculated levels of meHg in fish and seafood were 
shown in Table 1. Results showed significant differences $(P<0.05)$ between pelagic fish and the other three groups of demersal fish, crustacean and cephalopod $\left(\chi^{2}{ }_{K W}=49.090, p=0.000, N=405\right.$, Median $=$ $0.061 \pm 0.050 \mathrm{mg} / \mathrm{kg} \mathrm{WW})$. Concentrations of $\mathrm{meHg}$ in fish and seafood groups showed the highest in demersal fish $(0.1006 \mathrm{mg} / \mathrm{kg} \mathrm{WW})$ followed by in pelagic fish $(0.0686 \mathrm{mg} / \mathrm{kg} \mathrm{WW})$, freshwater fish $(0.045$ $\mathrm{mg} / \mathrm{kg} \mathrm{WW}$ ), cephalopods (0.0405 mg/kg WW) and crustaceans $(0.0356 \mathrm{mg} / \mathrm{kg} \mathrm{WW})$. Level of $\mathrm{meHg}$ reported in Algerian small pelagic fish, (sardine, Sardina pilchardus) $(0.04 \mathrm{mg} / \mathrm{kg} \mathrm{WW})$ is similar to levels from this study. However, in bigger pelagic (swordfish (Xiphias gladius); $0.57 \mathrm{mg} / \mathrm{kg} \mathrm{ww}$ ), its level is higher by nearly ten times (Mehouel et al 2019). Similarly, in Taiwan meHg level in swordfish was reported to be five times higher $(0.28 \mathrm{mg} / \mathrm{kg}$ of $\mathrm{meHg}$ ) (Hsi et al 2019). MeHg concentration in other most popular fish consumed by women of childbearing age in Taiwan, also showed higher levels compared to this study, excepted for species like mackerel, milkfish and anchovy. These group of researchers also reported that meHg levels in tilapia (Hsi et al 2019) is similar when compared to its level in freshwater catfish captured from this study. Tang and Chen with their co-workers $(2009,2014)$ respectively, reported on relatively low meHg levels in local small size farmed freshwater or marine whole fish from Hong Kong $(0.0045-0.16 \mu \mathrm{g} / \mathrm{g})$.

In this study, we also calculated fish and seafood consumption pattern by Malaysian population based on different background which inclusive four different age groups, three major ethnics and gender. Data on consumption of higher fish and seafood consumers were also calculated at three centiles of 75th, 90th and 95th. The main results illustrated the most relevant aspect of fish and seafood consumption patterns for the adolescence and adult population. In our previous fish consumption published data (Ahmad et al 2016), results were only presented for adults and discussion has emphasized on fish consumption frequencies or most consumed fish and seafood, most preferred cooking style, amounts of fish and seafood consumed by different types and groups, cooking style, and the amount per meal consumed by different ethnics in the country. Published data also described that Malay adolescent in this country consumed seafood most frequently compared to other food groups (Ahmad et al 2019). The consumption data together with levels of $\mathrm{meHg}$ in freshwater fish and seafood enables us to calculate and evaluate its contamination status and possible health risk in fish and seafood in Peninsular Malaysia. Table 6 indicated that the risk index (percentage of the PTWI) of meHg is not likely to cause health effects at the estimated mean of fish and seafood consumption at $89 \mathrm{~g} /$ day or $623 \mathrm{~g} /$ week (median data for overall fish and seafood consumption) using the JECFA PTWI value guideline. The risk index by different demographic factors were ranges between 30 to $51 \%$ of the PTWI. There were few studies attempt to compare fish and seafood consumption with dietary intakes estimates of meHg, and results were similar to this study where the risk index value was lower than the PTWI established by EFSA and JECFA (Mehouel et al 2019; Kuras et al 2017; Tang et al 2009; Tsuchiya et al 2008). Mehouel and coworkers (2019) assessed the risk of meHg intake through consumption of sardine and swordfish fished in three Algerian coasts, the EWI were at $2.8 \%$ and $40 \%$, respectively. They highlighted on the relationship between trophic levels and biomagnification factors where higher meHg concentration in large predatory fish is due to age, diet and time of exposure. An intervention research on intake of fish meals based in the Polish subpopulation also revealed the risk index level of up to $38.8 \%$ with range between 22.7 to $59.8 \%$.

Page $30 / 39$ 
These researchers also reported the hazard index at 0.39 and revealed that $32.8 \%$ of the volunteers exceeded the intake limit by the US-NRC $(0.7 \mu \mathrm{g} / \mathrm{kg} \mathrm{bw})$ at $800 \mathrm{~g} /$ week of fish consumption (Kuras et al 2017). Another related study is a dietary exposure of Hong Kong secondary school students which showed the estimated exposure to $\mathrm{meHg}$ at $25-31 \%$ for average fish consumers while the estimation for high consumer were between $75-88 \%$ of PTWI (Tang et al 2009). Tsuchiya and co-workers (2008) conducted a longitudinally study among women of child bearing age within the Japanese and Korean populations in the state of Washington, and reported on the differences between levels of total mercury intake by these two populations; the Japanese at $0.09 \mu \mathrm{g} / \mathrm{kg} / \mathrm{d}$ or $39 \%$ of PTWI and the Korean at 0.05 $\mu \mathrm{g} / \mathrm{kg} / \mathrm{d}$ or $22 \%$ of PTWI.

Our results also revealed that higher risk index for all demographic groups were contributed from the consumption of marine fish, specifically the demersal group. Despite these results, the highest risk index was showed from the overall consumption of seafood by the adolescent and contributed from the consumption of cephalopod (EWI $=0.8196 \mu \mathrm{g} / \mathrm{kg} \mathrm{BW} /$ week). For higher consumers, the EWI values were exceeded the PTWI (risk index $>100 \%$ ) which also contributed mainly from the consumption of marine fish. The minimum intake of $\mathrm{meHg}(0.0158$ to $0.3488 \mu \mathrm{g} / \mathrm{kg} \mathrm{BW} /$ week) was found from the consumption of crustacean in all demographic groups (risk index $<10 \%$ ) excepted at higher rate for the adolescent and Indian ethnicity, which is at 22 and $20 \%$, respectively. We also proved that meHg intake per kilogram body weight depended on species of fish and seafood being consumed. Exposure in some cases were close to the safety margin and observed in top predators and benthic carnivorous fish. Barone and co-workers (2015) also reported on this matter where among the highest risk index values calculated from the consumption of such group of fish, the example was from the consumption of European conger eel (1.26 $\mu \mathrm{g} / \mathrm{kg}$ bw/week), black belly rosefish (1.22 $\mu \mathrm{g} / \mathrm{kg}$ bw/week), long-nose skate (1.12 $\mu \mathrm{g} / \mathrm{kg} \mathrm{bw} /$ week), swordfish (1.44 $\mu \mathrm{g} / \mathrm{kg}$ bw/week), and Atlantic bluefin tuna (1.33 $\mu \mathrm{g} / \mathrm{kg}$ bw/week). Although the toxicological evaluation seems to be no important hazard, levels of meHg in these fishes should be under frequent surveillance. Suggestion have to be made for caution on their consumption by either regular fish consumers or the vulnerable groups of pregnant and lactating women, and also young children (Barone et al 2015).

In this current study, we pooled fish and seafood species into larger group and risk index were calculated per group, not for specific species. The present estimations were also consistent with recommended PTWI for general population and the emphasis is placed on the toxicity of meHg which is essentially accounts from the average data reported by Annual and co-workers (2018). If we considered worst-case situation on data for THg in fish and seafood samples, population with all demographic backgrounds would exceed the PTWI defined in the WHO/JECFA guidelines. As it is apparent from these results, a person from different demographic background can consumed 1 to $2 \mathrm{~kg}$ weekly of fish and seafood groups and still the PTWI established by the FAO/WHO will not be exceeded. However, for demersal fish, the same person can only consume $<1 \mathrm{~kg}(720 \mathrm{~g})$ weekly before exceeded the PTWI value limit of 1.6 $\mu \mathrm{g} / \mathrm{kg}$ bodyweight/week. The health risk exposure associated with the consumption of crustacean analyzed was minor with MSCW of $2 \mathrm{~kg}$ and above for all population groups. The exposure diet intake is linked to the HQ which signifies the relationship between the exposure obtained in the diet and the oral 
reference dose for meHg. The results of this study revealed health risk when HQs were computed for the vulnerable population in the community, while the HQ for adolescent and the elderly reached 1.1227 and 1.0489 , respectively. The HQ values close to 1 were associated with the consumption of fish and seafood for adults age $>41$ years old, Malay ethnic, both male and female group. Even, HQ for median fish and seafood consumption for overall population reached 0.8827 . The exposure to $\mathrm{meHg}$ in this study is likely to exceed the recommended value of PTWI in the case of consumption of high amount of fish and seafood with higher $\mathrm{Hg}$ content. Results showed that high consumers consumed fish and seafood at 322 to $406 \mathrm{~g} /$ day or 2.3 to $2.8 \mathrm{~kg} /$ week. The EWI would be 2.3 to $2.9 \mu \mathrm{g} / \mathrm{kg} \mathrm{BW} /$ week, or risk index of 146 to $184 \%$ compared to the PTWI. These factors caused HQ values to rise above one, ranging from 3.1 to 4.0 for more affluent consumers (fish/seafood intake in the 75th percentile). These results showed that average consumers are doubtful to encounter unnecessary health effects from meHg due to fish and seafood consumption, but the risk is higher for adolescent, the elderly and high consumers. This study also found that adolescent consumed different types of seafood compared to adults at different demographic factors. They preferred cephalopods and crustaceans and these groups contributed to the highest mean estimated meHg weekly intakes (EWI) other than from demersal fish. Although level of meHg in cephalopod and crustacean is lower compared to other marine fish, but high amount of consumption caused these groups of seafood were significant contributors to meHg accumulation in adolescent in Peninsular Malaysia. Similar patterns of results were reported elsewhere, for example Andrew et al., (2016) demonstrated the Disability Adjusted Life Years (DALYs) for eating different part of fish using a risk model (iRISK) and reported the frequency of consumption had exposed the children ( 17 years old) to non-carcinogenic risk, even the amount of $\mathrm{Hg}$ in fish part were minute. Their finding also showed that frequent access to tilapia fish in the community and district market, attributed to more DALY's. Other findings conducted among secondary school students in Hong Kong revealed that there were no undesirable health effects from consumption of median level of meHg of seafood for both average and high consumers but other sources of meHg namely shellfish and other seafood products might add significantly to dietary exposure (Tang et al 2009). Studies on the association between seafood consumption and meHg accumulation revealed higher average daily dose (ADD) level among higher seafood consumers whom resided in the coastal areas compared to the inland residents (Lee et al 2012; Jeevanaraj et al 2018).

\section{Conclusions}

The median concentrations of meHg in fish and seafood from Peninsular Malaysia were within the permissible limits by both national and international guidelines. MeHg evaluation seems to be no important hazard associated with average seafood consumers. However, the risk is significantly higher for high consumers when the value of EWI estimated for this group had approached the PTWI. Exposure in some cases was close to the safety margins thus, the meHg level in certain group of seafood; the demersal fish is recommended to be under frequent surveillance. Regular fish consumers are suggested to be caution in their consumption of seafood with higher levels of meHg, particularly young children and the elderly. There is a need for community compassion about risks associated with mercury especially for 
the vulnerable group. A potential exposure source from consumption of shellfish should be further monitored. There is also a need to investigate the amount of $\mathrm{meHg}$ in blood and hair for total population in the country as human biomonitoring programs are important tools in assessing current population exposure and in discovering trends and patterns related to policies, life style and food consumption. Information from this study is essential for assessing the effectiveness of policies and advisory authorities in developing relevant consumer recommendations with respect to consumption of seafood and health risk. There is a need to update and refine food consumption databases and levels of meHg in seafood for the purpose of constructing safe-eating guidelines to the public. Limitation of this study is meHg data in seafood were generated from an earlier study that reported from limited number of samples. Despite, this is the nearest estimated which is possible to calculate meHg data for risk assessment estimation to the population in the country.

\section{Declarations}

Author contribution Ahmad Nl: Conceptualization, methodology, sampling, sample and data analysis, visualization, preparation of the original draft, writing-review and editing. Wan Mahiyuddin WR:

Methodology and data analysis, Wan Azmi WNF: Methodology, sample and data analysis, Ruzman Azlee RS: Data analysis, Shaharudin R: Writing-review, Sulaiman LH: Conceptualization and methodology.

Funding Open access funding provided by JPP-IMR funds. This study was funded by the Ministry of Health Malaysia Research Grant (NMRR ID: 08-322-1477; JPP-IMR-07-025).

Data availability the datasets used and/or analyzed during the current study are available from the corresponding author on reasonable request.

Ethical approval the project was funded by the National Institutes of Health Malaysia, and the proposal was priory reviewed and approved by the Medical Research and Ethics Committee, Ministry of Health Malaysia.

Consent of participate the inform consent and confidentiality was obtained from the subjects included in the study beforehand

Consent for publication the consent for publication was obtained from a desk of Director General of Health Malaysia. A special thanks to the Director General of Health Malaysia for giving permission to publish this article.

Competing interest the authors declare that there is no competing interest.

Acknowledgements the authors acknowledge support and assistance provided by staffs of the Environmental Health Research Center, Institute for Medical Research; Fisheries Development Authority of Malaysia, and Fish Quality Control Center, Department of Fisheries. 


\section{References}

1. Agusa T, Kunito T, Yasunaga G, Iwata H, Subramanian A, Ismail A, Tanabe S. (2005). Concentration of trace elements in marine fish and its risk assessment in Malaysia. Marine Pollution Bulletin. 51:896-911. Doi:10.1016/j/m1rpolbul.2005.06.007.

2. Ahmad NI. (2007). Penilaian risiko pengambilan sisa racun perosak terpilih melalui pemakanan sayur-sayuran di kalangan penduduk dewasa di negeri Selangor. Malaysia: Universiti Kebangsaan Malaysia:PhD thesis. https://malcat.uum.edu.my/kip/Record/ukm..b14173888

3. Ahmad NI, Wan Rozita W.M, Mohd Fairulnizal M.N., Tengku Rozaina T.M. ${ }^{*}$, Zarina Z. ${ }^{*}$, Hamdan J ${ }^{\star \star}$, Siti Fatimah D. \& Suraya Z. (2011). The prevalence of overweight and obesity and its associated factors among students aged 10-17 years old: findings from the seafood consumption survey in Peninsular Malaysia, 2008-2009. MASO 2011 Scientific Conference on Obesity-Towards healthy weight for life. 28-29 June. Kuala Lumpur. Abstract pg 84. Available from: http://www.maso.org.my/download/MASO\%202011\%20souvenir\%20program.pdf

4. Ahmad NI, Mohd Fairulnizal MN, Wan Rozita WM,Hamdan J, Ismail I, Wan Nurul Farah WA, Yuvaneswary V, Mohd Hairulhisam H. (2015 $)$. Mercury levels of marine fish commonly consumed in Peninsular Malaysia. Environmental Science and Pollution Research. 22:3672-3686. DOI 10.1007/s11356-014-3538-8.

5. Ahmad NI, Mohd Fairulnizal M.N., Wan Rozita W., Hamdan J, Ismail I, Wan Nurul Farah W. A., Yuvaneswary V. and Fazlin Anis M. (2015 $)$ Determination of total mercury levels in commercial cephalopod and crustacean in Peninsular Malaysia. Environmental Science and Pollution Research. DOI 10.1007/s11356-015-4415-9

6. Ahmad NI, Wan Rozita WM, Tengku Rozaina TM, Cheong YL, Siti Fatimah D, Nasriyah CH, Nor Aini A, Rafiza S, Lokman Hakim S. (2016). Fish consumption pattern among adults of different ethnics in Peninsular Malaysia. Food \& Nutrition Research. 60:32697-http://dx.doi.org/10.3402/fnr.v60.32697.

7. Ahmad NI, Nadia M, Wan Rozita WM, Tengku Rozaina TM, Rafiza S, Lokman Hakim S. (2019). The Prevalence of Overweight and Obesity and Its Association Factors among Malays' Adolescents: Findings from Seafood Consumption Survey in Peninsular Malaysia. Journal of Childhood Obesity 4:2. http://childhood-obesity.imedpub.com.

8. Alina M, Azrina A, Mohd Yunos AS, Mohd Zakiuddin S, Mohd Izuan Effendi H, Muhammad Rizal R (2012) Heavy metal (mercury, arsenic, cadmium, plumbing) in selected marine fish and shellfish along the Straits of Malacca. International Food Research Journal 19(1):135-140 doi: 10.1016/j.marpolbul.2005.06.007.

9. Al-Majed NB \& Preston MR. (2000). An assessment of the total and methyl mercury content of zooplankton and fish tissue collected from Kuwait Territorial Waters. Marine Pollution Bulletin; 2000:40(4):298-307. https://doi.org/10.1016/S0025-326X (99)00217-9.

10. Al-Mughairi S, Yesudhason P, AL-Busaidi M, AL-Waili A, Al-Rahbi WAK, Al-Mazrooei N, Al-Habsi SH. (2013). Concentration and exposure assessment of mercury in commercial fish and other seafood 
marketed in Oman. Journal of Food Science; 78(7). T1082-T1090. https://doi.org/10.1111/17503841.12150

11. Andrew T, Francis E, Charles M, Irene N, Jesco N, Ocaido M, Drago K, Celcus S, Deborah A, Rumbeiha W. (2016). Risk estimates for children and pregnant women exposed to mercury-contmainated Oroechromis niloticus and Lates niloticus in Lakes Albert Uganda. Cogent Food \& Agriculture; 2:1228732. Hppt://dx.doi.org/10.1080/23311932.2016.1228732.

12. Annual ZF, Maher W, Krikowa K, Sulaiman LH, Ahmad NI, Foster S. (2018). Mercury and risk assessment from consumption of crustaceans, cephalopods and fish from West Peninsular Malaysia. Microchemical Journal. 140:214-221. https://doi.org/10.1016/j.microc.2018.04.024

13. Barone G, Storelli R, Busco VP, Quaglia NC, Centrone G, Storelli MM. (2015). Assessment of mercury and cadmium via seafood consumption in Italy: estimated dietary intake (EWI) and target hazard quotient (THQ). Food Additives \& Contaminants:Part A. DOI:10.1080/19440049.2015.1055594.

14. Bhupander K \& Mukherjee DP. (2011). Assessment of human health risk for arsenic, copper, nickel and zinc in fish collected from Tropical Wetlands in India. Advances in Life Science \& Technology. ISSN 2224-7181 (Paper) ISSN 2225-062X Vol 2.

15. Budiyanto F, Arbi UY, Suratno. (2019). Risk assessment on mercury concentration in six edible mollusks from Bintan Island, Indonesia. Inter. Conference on Biology and Applied Science (ICOBAS). AIP Conf. 2120, 040009-1-040009-8; https://doi.org/10.1063/1.5115647.

16. Burger J. (2009). Risk to consumers from mercury in bluefish (Pomatomus saltatrix) from New Jersey: size, season and geographical effects. Environmental Research. 109:803-811 https://doi.org/10.1016/j.envres.2009.07.005

17. Castro-Gonžalez MI, Mendez-Armenta M. (2008). Heavy metal: implications association to fish consumption. Environmental Toxicology and Pharmacology, 26:263-271 doi: 10.1016/j.etap.2008.06.001.

18. Chen MYY, Wong WWK, Chung SWC, Tran CH, Chan BTP, Ho YY, Xiao Y. (2014). Quantitative riskbenefit analysis of fish consumption for women of child-bearing age in Hong Kong. Food Additives \& Contaminants. Part A. 31(1): 48-53. doi: 10.1080/19440049.2013.855947

19. Chen YC, Chen MH (2006). Mercury levels of seafood commonly consumed in Taiwan. Journal of Food and Drug Analysis; 14(4):373-378 https://doi.org/10.38212/2224-6614.2450

20. Cheng J, Gao L, Zhao W, Liu X, Sakamoto M \& Wang W. (2009). Mercury levels in fishermen and their household members in Zhoushan, China: Impact of public health. Science of Total Environment. 407:2625-2630.

21. Chouvelon T, Warnau M, Churlaud C, Bustamante $P$ (2009) Hg concentrations and related risk assessment in coral reef crustaceans, mollusk and fish from New Caledonia. Environmental Pollution; 157:331-340. https://doi.org/10.1016/j.envpol.2008.06.027.

22. Clarkson TW, Magos L, Myers GJ (2003) The toxicology of mercury-current exposures and clinical manifestations. New England Journal of Medicine 349:1731-1737 doi: 10.1056/NEJMra022471. 
23. de Matos LS, Otavio J, Silva S, Kasper D, Carvalho LN. (2018). Assessment of mercury contamination in Brycon falcatus (Characiformes: Bryconidae) and human health risk by consumption of this fish from the Teles Pires River, Southern Amazonia. Neotropical Ichthyology. 16(1): e160106. DOI: 10.1590/1982-0224-20160106

24. FAO/WHO Evaluation of certain food additives and contaminants, (2006). Sixty-seventh report of the Joint FAO/WHO Expert Committee on Food Additives. WHO technical report series. no 940. Retrieved January 2019 from https://apps.who.int/iris/handle/10665/43592

25. Hajeb P, Jinap S. (2011). Mercury exposure through fish and seafood consumption in the rural and urban coastal communities of Peninsular Malaysia. World Journal Fish Marine Science 3(3):217226.

26. Hajeb P, Jinap S, Ismail A, Fatimah AB, Jamilah B, Abdul Rahim M. (2009). Assessment of mercury level in commonly consumed marine fishes in Malaysia. Food Control. 20:79-84. doi: 10.1016/j.foodcont.2008.02.012.

27. Hsi HC, Hsu YW, Chang TC, Chien LC. (2016). Methylmercury concentration in fish and risk-benefit assessment of fish intake among pregnant versus infertile women in Taiwan. Plos One. DOI:10.1371/journal.pone.0155704.

28. Jeevanaraj P, Hashim Z, Elias SM, Aris AZ. (2018). Risk of dietary mercury exposure via marine fish ingestion: Assessment among potential mothers in Malaysia, Exposure Health. https://doi.org/10.1007s12403-017-0270-x.

29. Juric AK, Batal M, David W, Sharp D, Schwartz H, Ing A, Fediuk K, Black A, Tikhonov C, Chan LHM. (2017). A total diet study and probabilistic assessment risk assessment of dietary mercury exposure among First Nations living on-reserve in Ontario, Canada. Environmental Research. 158:409-420. doi: 10.1016/j.envres.2017.06.025.

30. Kamaruzaman BY, Rina Z, John BA, Jalal KCA (2011). Heavy metal accumulation in commercial important fishes of South West Malaysian Coast. Research Journal of Environmental Sciences;1-8. https://dx.doi.org/10.3923/rjes.2011.595.602

31. Khaniki GRJ, Alli I, Nowroozi E, Nabizadeh R (2005) Mercury contamination in fish and public health aspects: a review. Pakistan Journal of Nutrition 4(5): 276-281 https://dx.doi.org/10.3923/pjn.2005.276.281

32. Kuras R, Janasik B, Stanislawska M, Kozlowska L, Wasowicz W. (2017). Assessment of mercury intake from fish meals based in Intervention Research in the Polish Subpopulation. Biological Trace Element Research. 179:23-31. DOI 10.1007/s12011-017-0939-9.

33. Larsen R, Eilertsen KE, Elvevoll EO (2011). Health benefits of marine foods and ingredients. Biotechnology Advances. 29:508-518 doi: 10.1016/j.biotechadv.2011.05.017.

34. Lee SH., Cho YH., Park SO, Kye SH, Kim BH, Hahm TS, Kim M, Lee JO \& Kim Cl. (2006). Dietary exposure of the Korean population to arsenic, cadmium, lead and mercury. Journal of Food Composition and Analysis; 19: S31-S37. https://doi.org/10.1016/j.jfca.2005.10.006 
35. Low KH, Zain SM, Abas MR, Salleh KM, Teo YY. (2015). Distribution and health risk assessment of trace metals in freshwater tilapia from three different aquaculture sites in Jelebu Region (Malaysia). Food Chemistry. 390-396. doi: 10.1016/j.foodchem.2015.01.059.

36. Malaysian Food Regulation 1985. International Law Book Services (2006) Food act 1983 (Act 281) \& regulations-laws of Malaysia. Petaling Jaya, Selangor.

37. McManus A, Feilder L, Newton W, White J. (2011). Health benefits of seafood for men. Journal of Men's Health. 8(4):252-257 https://doi.org/10.1016/j.jomh.2011.04.004

38. Mehouel F, Bouayad L, Berber A, Van Hauteghem I, Van de Wiele. (2019). Risk assessment of mercury and methyl mercury intake via sardine and swordfish consumption in Algeria. Journal of Hellenic Veterinary Medical Society. 70(3):1679-1686. https://doi.org/10.12681/jhvms.21792

39. Mohd Fairulnizal MN, Tumijah AH, Zakiah I. (1998). Determination of mercury in urine by on-line digestion with a flow injection mercury system. Atomic Spectroscopy. 19(3):95-99. https://www.researchgate.net/publication/232804981_Determination_of_Mercury_in_Urine_by_OnLine_Digestion_with_a_Flow_Injection_Mercury_System

40. Mok WJ, Senoo S, Itoh T, Tsukamasa Y, Kawasaki K, Ando M. (2012). Assessment of concentrations toxic elements in aquaculture food products in Malaysia. Food Chemistry, 133:1326-1332 https://doi.org/10.1016/j.foodchem.2012.02.011

41. Morgano MA, Rabonato LC, Milani RF, Miyagusku L, Balian SC (2011) Assessment of trace elements in fishes of Japanese foods marketed in Sao Paulo (Brazil). Food Control 22:778-785 https://doi.org/10.1016/j.foodcont.2010.11.016.

42. Myers GJ, Davidson PW. (2000). Does methyl mercury have a role in causing development disabilities in children? Environmental Health Perspectives. 108(3):413-420 https://dx.doi.org/10.1289\%2Fehp.00108s3413

43. Nurnadia AA, Azrina A, Amin I. (2011). Proximate composition and energetic value of selected marine fish and shellfish from the West Coast of Peninsular Malaysia. International Food Research Journal; 18:137-148. http://www.ifrj.upm.edu.my/18\%20(01)\%202011/(14)\%20IFRJ-2010059\%20Azrina\%20UPM[1].pdf.

44. Ouboter PE, Landburg G, Satnarain GU, Starke SY, Nanden I, Simon-Friedt B, Hawkins WB, Taylor R, Lichtveld MY, Harville E, Wickliffe JK. (2018). Mercury levels in Women and children from interior villages in Suriname, South America. International Journal of Environmental Research and Public Health. 15:1007. Doi:10.3390/ijerph15051007.

45. Saei-Dehkordi SS, Fallah AA \& Nematollahi A. (2010). Arsenic and mercury in commercially valuable fish species from the Persian Gulf: Influence of season and habitat. Food Chemistry and Toxicology. 48:2945-2950. https://doi.org/10.1016/j.fct.2010.07.031

46. Sioen I, Matthys C, De Backer G, Van Camp J, Henauw SD. (2007). Importance of seafood as nutrient source in the diet of Belgian adolescents. The Journal of Human Nutrition and Dietetics 20: 580_6. doi: http://dx.doi.org/10.1111/j.1365-277X.2007.00814.x 
47. Statistical Department of Malaysia 2001. (2000). Press Statement: Population distribution and basic demographic characteristics report population and housing census. Available from: http://www.statistics.gov.my/English/pressdemo.htm. [Cited 10 July 2015].

48. Stuchal LD, Charles-Ayinde MKS, Kane AS, Kozuch M, Roberts SM. (2019). Probabilistic risk assessment for high-end consumers of seafood on the Northeastern Gulf Coast. Journal of Exposure Science \& Environmental Epidemiology. Author manuscript; available in PMC. August 07. https://dx.doi.org/10.1038\%2Fs41370-019-0119-4

49. Suratno, Puspitasari R, Rositasari R, Oktaviyani S. (2019). Total mercury of marine fishes in Natuna Islands area, Indonesia: Risk assessment for human consumption. $3^{\text {rd }}$ International Symposium on Green Technology for value Chains 2018. IOP Publishing. IOP Conf Series: earth and Environmental Science 277 012025, Doi:10.1088/1755-1315/277/1/012025.

50. Suzana, S., Rafidah, G., Noor Aini, M.Y., Nik Shanita, S., Zahara, A.M. and Shahrul Azman, M.N. (2002). Atlas Makanan: Saiz pertukaran dan Porsi. Universiti Kebangsaan Malaysia. Kuala Lumpur: MDC Publishers \& Printers Sdn Bhd.

51. Suzana, S., Noor Aini, M.Y., Nik Shanita, S., Rafidah, G. and Roslina A. (2009). Atlas Makanan: Saiz pertukaran dan Porsi. (second edition) Universiti Kebangsaan Malaysia. Kuala Lumpur: MDC Publishers \& Printers Sdn Bhd.

52. Tang ASP, Kwong KP, Chung SWC, Ho YY, Xiao Y. (2009). Dietary exposure of Hong Kong secondary school students to total mercury and methylmercury from fish intake. Food Additives \& Contaminants. Part B; 2(1):8-14. https://doi.org/10.1080/02652030802642102

53. Tee, E.S., Mohd Ismail, N., Mohd Nasir, A. \& Khatijah, I. (1997). Nutrient composition of Malaysian foods. ASEAN Sub-Committee on protein: Food habits research and development, Kuala Lumpur: Institute for Medical Research.

54. Tsuchiya A, Hinners TA, Burbacher TM, Faustman EM, Marien K. (2008). Mercury exposure from fish consumption within the Japanese and Korean communities. Journal of Toxicology and Environmental Health, Part A, 71:1019-1031. https://doi.org/10.1080/01932690801934612

55. US Environmental Protection Agency (USEPA) (2000). Guidance for assessing chemical contaminant data for use in fish advisory vol. II: Risk assessment and fish consumption limits. US Environmental Protection Agency. Office of Science and Technology. Office of Water, Washington (D.C.), EPA823-B00-008. Retrieved January 2019 from https://www.epa.gov/sites/default/files/201506/documents/volume2.pdf

56. von Stackelberg K, Li Miling, Sunderland E. (2017). Results of a national survey of high-frequency fish consumers in the United States. Environmental Research. 158:126-136. http://dx.doi.org/10.1016/j.envres.2017.05.042

57. Wan Azmi WNF, Ahmad NI, and Wan Mahiyuddin WR. (2019). Heavy metal levels and risk assessment from consumption of marine fish in Peninsular Malaysia. Journal of Environmental Protection. 10(11). https://doi.org/10.4236/jep.2019.1011086 
58. WHO (2004). Evaluation of certain food additives and contaminants. Sixty-First Report of the Joint FAO/WHO Expert Committee on Food Additives. Retrieved January 2019 from https://apps.who.int/iris/handle/10665/42849

59. WHO/FAO (2011). Safety evaluation of certain contaminants in food. WHO Food Additive. Series 63, India p. 673 Retrived January 2019 from http://www.fao.org/3/at881e/at881e.pdf

60. Whyte ALH, Hook GR, Greening GE, Gibbs-Smith E, Gardner JPA. (2009). Human dietary exposure to heavy metals via the consumption of greenshell mussels (Perna canaliculus Gmelin 1791) from the Bay of Islands, Northern New Zealand. Science of the Total Environment. 407:4348-4355. https://doi.org/10.1016/j.scitotenv.2009.04.011

61. You SH, Wang SL, Pan WH, Chan WC, Fan AM, Lin P. (2018). Risk assessment or methyl mercury based on internal exposure and fish and seafood consumption estimates in Taiwanese children. International Journal of Hygiene and Environmental Health. hyyps://doi.org/10.1016/j.ijeh.2018.03.220.

62. Zolfaghari G. (2018). Risk assessment of mercury and lead in fish species from Iranian international wetlands. Methods X. 5:438-447. https://doi.org/10.1016/j.mex.2018.05.002 